\title{
Stress-augmented thermal activation: Tribology feels the force
}

\author{
Hugh SPIKES \\ Tribology Group, Department of Mechanical Engineering, Imperial College, London SW72BX, UK \\ Received: 22 September 2017 / Accepted: 24 November 2017 \\ (C) The author(s) 2018. This article is published with open access at Springerlink.com
}

\begin{abstract}
In stress-augmented thermal activation, the activation energy barrier that controls the rate of atomic and molecular processes is reduced by the application of stress, with the result that the rate of these processes increases exponentially with applied stress. This concept has particular relevance to Tribology, and since its development in the early twentieth century, it has been applied to develop important models of plastic flow, sliding friction, rheology, wear, and tribochemistry. This paper reviews the development of stress-augmented thermal activation and its application to all of these areas of Tribology. The strengths and limitations of the approach are then discussed and future directions considered. From the scientific point of view, the concept of stress-augmented thermal activation is important since it enables the development of models that describe macroscale tribological performance, such as friction coefficient or tribofilm formation, in terms of the structure and behaviour of individual atoms and molecules. This both helps us understand these processes at a fundamental level and also provides tools for the informed design of lubricants and surfaces.
\end{abstract}

Keywords: stress activation; stress augmented thermal activation; mechanochemistry; friction; EHD friction; wear; Eyring

\section{Introduction}

Tribology is concerned with the interaction of surfaces in relative motion and the behaviour of interfaces present between these surfaces. Such relative motion is produced by applied shear forces and is opposed by friction.

Despite more than a hundred years of research we still have surprisingly little deep understanding of how the applied forces, the friction, and the relative motion are connected at an atomic and molecular scale. Most tribological experiments are carried out at the macroscale, where, while they provide useful relationships between friction, load, temperature, and sliding velocity, they do not reveal whence these originate. Computer-based, molecular-scale simulations are starting to provide some insights and are certainly the way forward but they have yet to be linked fully to the macroscale laws of friction and sliding derived from experiment.
There is, however, one model or group of models that is able to connect directly molecular-scale behaviour to macroscale friction response and other tribological phenomena. This model was developed independently in quite different contexts by Prandtl between 1913 and 1928 [1,2] and Eyring in 1936 [3]. It is based on the concept that a mechanical force applied to an atom or molecule couples with the thermal energy possessed by this entity to increase the rate of a process. This process may be sliding, fluid flow or chemical reaction, or indeed any process that involves an atom or molecule traversing an energy barrier. Because it involves a combination of mechanical force and thermal energy this concept is sometimes called stress-promoted or stress-augmented thermal activation but no standard descriptor has yet become widely accepted.

The essential idea starts with the Arrhenius rate equation in which the rate constant $k_{\mathrm{o}}$ is given by:

$$
k_{\mathrm{o}}=A \mathrm{e}^{-E_{\mathrm{a}} / k_{\mathrm{B}} T}
$$

* Corresponding author: Hugh SPIKES, E-mail: h.spikes@imperial.ac.uk 
where $A$ is a pre-constant, $E_{\mathrm{a}}$ is the thermal activation energy for the process, $k_{\mathrm{B}}$ is Boltzmann's constant, and $T$ is the absolute temperature. This equation can be quite easy understood in terms of probability since according to the Boltzmann energy distribution, $\mathrm{e}^{-E_{\mathrm{a}} / k_{\mathrm{B}} T}$ is the fraction of atoms or molecules in a large ensemble that have energy greater than $E_{a}$ and thus enough to traverse this energy barrier. It can however also be derived from thermodynamic or statistical mechanics principles with $E_{\mathrm{a}}$ replaced by a free energy [4].

According to stress-augmented thermal activation, when a force, $f$, is applied to the atom or molecule along the direction of the process, Eq. (1) is modified to

$$
k_{\mathrm{f}}=A \mathrm{e}^{-\left(E_{\mathrm{a}}-f \Delta x\right) / k_{\mathrm{B}} T}
$$

where $\Delta x$ is the distance the force must move the atom or molecule to reach the top of the energy barrier (the activation length). The term $f \Delta x$ is a work term and has the effect of reducing the energy barrier and so increasing the rate of the process. The impact of this term on the rate can easily be seen from the rearrangement

$$
k_{\mathrm{f}}=A \mathrm{e}^{-E_{\mathrm{a}} / k_{\mathrm{B}} T} \mathrm{e}^{+f \Delta x / k_{\mathrm{B}} T}
$$

where it is evident that the rate of the process increases exponentially with the applied force. It should be noted that Eq. (2) is the simplest model of stressaugmented thermal activation and this has been extended and embellished to a considerable degree over the years, as will be discussed later in this paper. This equation and those that follow can, of course, be scaled up from molecular to molar quantities so that

$$
k_{\mathrm{f}}=A \mathrm{e}^{-E / R T} \mathrm{e}^{+N f \Delta x / R T}
$$

where $E$ is now the molar thermal activation energy, $N$ is Avogadro's number, and $R$ the gas constant.

In Eqs. (2) and (3), $k_{\mathrm{f}}$ is the forward rate constant of the process, i.e., in the direction of the applied force. The applied force also reduces the backward rate of reaction according to

$$
k_{\mathrm{b}}=A \mathrm{e}^{-\left(E_{\mathrm{a}}+f \Delta x\right) / k_{\mathrm{B}} T}
$$

so that in systems where both forward and reverse processes are possible and have the same prefactor, the overall net reaction rate constant becomes

$$
k_{\text {net }}=A\left(\mathrm{e}^{-\left(E_{\mathrm{a}}-f \Delta x\right) / k_{\mathrm{B}} T}-\mathrm{e}^{-\left(E_{\mathrm{a}}+f \Delta x\right) / k_{\mathrm{B}} T}\right)
$$

Upon rearrangement this is

$k_{\text {net }}=A \mathrm{e}^{-E_{\mathrm{a}} / k_{\mathrm{B}} T}\left(\mathrm{e}^{f \Delta x / k_{\mathrm{B}} T}-\mathrm{e}^{-f \Delta x / k_{\mathrm{B}} T}\right)=2 k_{\mathrm{o}} \sin h\left(f \Delta x / k_{\mathrm{B}} T\right)$

At high applied forces or when no reverse process is possible the second term is negligible, in which case the rate of a process will vary exponentially with the applied force.

The influence of the applied force on the energy barrier is shown schematically in Fig. 1 for a very simple case where, prior to the application of force, the height of the energy barrier is the same in forward and reverse directions.

The above model has been applied to many disciplines including various aspects of tribology, without, however, ever becoming central to the latter. In the author's view it is surprising since the concept appears uniquely relevant to tribology, which is concerned the kinetic response of systems to large applied mechanical forces.

The aim of this paper is therefore to show how this stress-augmented thermal activation principle applies to Tribology, to raise its general profile as a key descriptor of many tribological processes and so help understand these processes. The paper first provides a brief history of how the model was developed and applied in various forms to different areas of tribology up to the 1950s. This is then followed by a more detailed review of each of the most significant areas of application, plastic deformation, lubricant rheology,

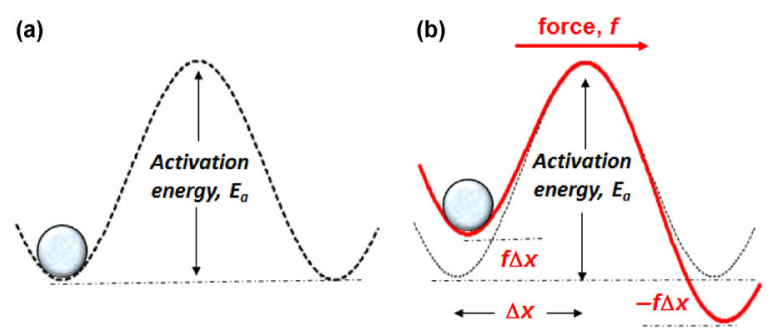

Fig. 1 (a) Activation energy barrier in the absence of an applied force on the molecule shown. (b) Influence of an applied force $f$ on the effective energy barrier. Adapted and reproduced with permission from [5]. Copyright AIP, 1938. 
sliding friction, tribochemistry, and wear. Finally, there is a general discussion that attempts to bring together the various ways that stress-augmented thermal activation has been applied across Tribology and to look forward to future developments.

\section{Early history}

The concept of stress-augmented thermal activation appears to have been first developed by Ludwig Prandtl at the University of Göttingen in the early 1900s, being outlined by his student von Karman in 1913 [1] and described in full by Prandtl himself in 1928 [2]. Prandtl's interest was in crystal plasticity and, as described in the next section, he derived an expression for the rate of plastic flow of a metal in terms of the effect of applied stress on the forward and reverse motion of atoms across the potential field of an adjacent atomic plane. This gave an expression quite similar to Eq. (6) above. Although Prandtl's focus was on metal deformation he noted at the end of his 1928 paper:

"To conclude, it may now be suggested that our conceptual model is also suitable for the treatment of kinetic friction."

Prandtl's model and a related one by Becker [6] was applied in a series of subsequent studies of metal deformation to describe dislocation motion, creep, and plastic flow, as described later in this paper. However, it did not appear to reach the attention of researchers in the fields of friction, lubrication, and wear until the early 2000s.

In 1936 Henry Eyring at Princeton developed a model of liquid viscosity using a similar concept [3]. Since his ideas originated from his work on chemical kinetics he described his model as being based on absolute reaction rates. For simple molecular liquids he envisaged liquid flow as originating from the movement of individual molecules over the potential field of an adjacent plane of molecules. In the absence of an applied shear stress there will be no net flow since the forward and reverse rate constants of flow will be the same. However, from Eq. (7) an applied shear stress will impose a force on the molecules so as to produce the difference in the velocity between adjacent layers of the form

$$
\Delta u=2 k_{\mathrm{o}} \lambda \sin h\left(\frac{f \lambda}{2 k_{\mathrm{B}} T}\right)
$$

where $\lambda / 2$ is the distance the molecules move from the bottom to the top of the energy barrier and is thus equivalent to the activation length $\Delta x$ in Eqs. (2)-(7) and Fig. 1. As described later in this review, Eyring converted this velocity difference to a shear rate and the force to a shear stress and thus derived a rheological equation relating shear rate and shear stress. At moderate shear stress the shear rate varied with the $\sin h()$ of the shear stress, but at high shear stress this simplified to an exponential dependence.

Although Eyring developed his equation in terms of individual molecular motion, he was well aware of its general thermodynamic origins. Thus he wrote in 1941 [7]:

"The general equation giving the rate of any reaction which has been modified by some external agency may be written:

$$
k^{\prime}=\left(\frac{k_{\mathrm{B}} T}{h}\right) \mathrm{e}^{\left(-\Delta F^{\ddagger} / R T-\Delta F^{\ddagger} / R T\right)}
$$

Here $\Delta F^{f^{\prime}}$ is the contribution made by the external agency to the free energy of activation."

Eyring's external agency was of course applied force. Eyring and his students went on to apply this model to the prediction of the viscosities of simple liquids $[5,8,9]$, polymer solutions $[10,11]$, and unimolecular films [12], to the rate of deformation of metals [13], and to the flow and creep of polymers [14, 15]. In the latter he treated flow as a balance between the breaking and reforming of molecular bonds and noted the potential applicability of this to tribology, writing [14]:

"The phenomena of friction and lubrication appear to involve this type of equilibrium between rate of breaking and re-formation of bonds."

There is no evidence that Eyring was aware of Prandtl's earlier work and indeed the two models originate from very different starting points, Prandtl's based on mechanical principles and Eyring's on chemical kinetics. A recent paper has compared the two approaches and the similarities and differences between them in some detail, as well as briefly outlining the general application of stress-augmented thermal activation to Tribology [16]. 
In 1953, Schallamach studied the kinetic friction of rubber sliding against ground glass and silicon carbide paper and found that the logarithm of the sliding velocity, $v$, varied linearly with the applied force, $F$, and inversely with the absolute temperature $[17,18]$. Based on Eyring's approach and assuming negligible reverse process he proposed an equation relating sliding velocity, $v$, to force, $F$, and temperature, $T$, very similar to Eq. (3)

$$
v=A \mathrm{e}^{-(E-\gamma F) / k_{\mathrm{B}} T}
$$

where $E$ is an activation energy and $A$ and $\gamma$ are constants, the latter being the activation length. Clearly this equation predicts that $F$ will be proportional to $\log (v)$ at constant temperature.

In the 1950s, Beuche developed a model to describe the mechanical scission of polymers under shear. His model was initially based on a combined effect of thermal and stored elastic energy in breaking polymer bonds [19], but this was soon modified to one very similar to Eyring's, with probability of bond breaking, $p$, given by

$$
p=\mathrm{e}^{-(E-F \mathcal{S}) / k_{\mathrm{B}} T}
$$

where $F$ is the tension in the bond and $2 \delta$ the distance the bond will stretch before breaking [20, 21].

Also in the 1950s, Zhurkov investigated lifetime to fracture in uniaxial tension of a wide range of solid materials including metals, ceramics, and polymers [22-24] and found a dependence on stress and temperature of the form

$$
\tau=\tau_{\mathrm{o}} \mathrm{e}^{\left(u_{\mathrm{o}}-\gamma \sigma\right) / k_{\mathrm{B}} T}
$$

where $\tau$ is the lifetime, $U_{\mathrm{o}}$ an activation energy for bond dissociation, $\sigma$ the applied tensile stress, and $\tau_{\mathrm{o}}$ and $\gamma$ are constants. The similarity of this to Eyring's model is evident, with the exponent in Eq. (12) being positive simply because $\tau$ is a lifetime, and thus reciprocal to a rate. Another difference from Eqs. (8), (10), and (11) is that the work term in Eq. (12), $\gamma \sigma$, is based on applied shear stress rather than applied force, so that the activation constant $\gamma$ has units of volume rather than length; this difference will be discussed later in this paper. It is not clear whether Beuche or Zhurkov were aware in the 1950s of Eyring's work, though in later papers Zhurkov does reference Eyring's work on polymer flow [24, 25].

From the above it can be seen that, by the end of the 1950s, the stress-augmented thermal activation approach pioneered by Prandtl and Eyring had been successfully used in models of plastic deformation, liquid viscosity, kinetic friction, and chemical bond dissociation.

In subsequent years these models have been further applied and developed and, as described below, have helped us understand the atomic and molecular origins of key tribological processes. The next five sections of this paper will illustrate this by reviewing, in turn, the application of stress-augmented thermal activation models to five areas of relevance to tribology: (i) plastic deformation of materials, (ii) lubricant rheology, (iii) sliding friction, (iv) tribochemistry, and (v) wear.

\section{Plastic deformation}

\subsection{Prandtl's model}

Prandtl's concept of stress-augmented thermal activation was first described fully in 1928 [2]. Prandtl considered the forces involved when a single, elastically constrained metal atom slides across the sinudoidal force field produced by a stationary plane of adjacent atoms, as shown schematically in Fig. 2(a). A plot of the force experienced by this atom as it traverses the stationary surface is shown in Fig. 2(b). The rest position of the atom is at $C$ but its actual position is at $D$ where the (negative) linear, elastic, constraining force shown as straight line, intercepts and balances the sinusoidal force field at force $\mathrm{E}$. As the atom moves to the right it tracks the sinusoidal force field until, so long as it is at absolute zero temperature, it reaches position $\mathrm{D}^{\prime}$ where the force is $E^{\prime}$. Here it becomes unstable and jumps to the alternative intercept position $\mathrm{D}^{\prime \prime}$, corresponding to force $E^{\prime \prime}$. If the motion is now reversed the atom does not jump back to $\mathrm{D}^{\prime}$ but instead initially tracks the sinusoidal force field and Prandtl explained the hysteresis of plastic deformation process on the basis of this irreversible process. This concept is now known as the Prandtl-Tomlinson model [16].

However, in practice the atom will be at a temperature considerable above absolute zero and so will possess thermal energy. This means that it will 
be able to jump sooner than from $\mathrm{D}$, for example from $\mathrm{P}$, when the force is $\mathrm{Q}$, to $\mathrm{P}^{\prime}$. To do this it must possess thermal energy $U_{1}$, which corresponds to the horizontally hatched area in Fig. 2(b). The reverse process is much less likely since the atom needs energy $U_{2}$, corresponding to the vertically-hatched area, in order to jump from $\mathrm{P}^{\prime}$ to $\mathrm{P}$.

Figure 2(b) shows the variation of force with $x$, the coordinate of the stationary surface, while Fig. 2(c) shows this force as a function of the displacement of the frame (or plane of atoms) in which the single atom is elastically constrained, $\xi$. If it possesses the requisite thermal energy, the atom jumps from force Q to $Q^{\prime}$ spontaneously at a fixed position of the sliding frame. Indeed, depending on the thermal energy available this jump can take place anywhere between $-a$ and $a$.

Prandtl then considered a very large number of such atoms, irregularly distributed in the sliding frame and non-commensurate with the adjacent force field. The proportion of atoms at a particular location $\xi$ between $-a$ and $a$ in the upper part of the curve in Fig. 2(c) (and thus available to jump down) is $\mu$. The fraction of these atoms having energy $U_{1}$ sufficient to jump is given by the Arrhenius expression $\mathrm{e}^{-U_{1} / k_{\mathrm{B}} T}$. The corresponding proportion of atoms in the lower part of the curve is $1-\mu$ and the fraction with enough energy to jump upward is $\mathrm{e}^{-u_{2} / k_{\mathrm{B}} T}$. Thus Prandtl derived the net rate at which atoms would move to the upper part of the curve as

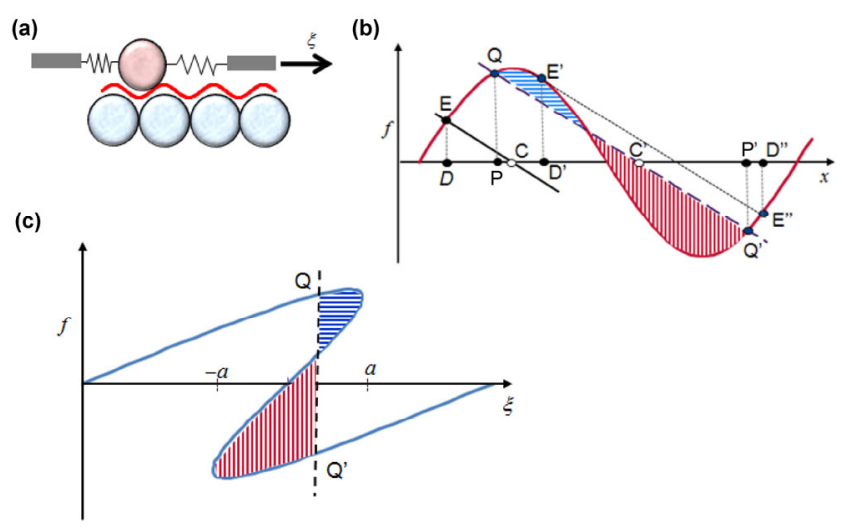

Fig. 2 (a) Basis of Prandtl model of an elastically constrained atom sliding over a plane of atoms; (b) force plotted against stationary surface coordinate showing instability at D'; (c) force plotted against slider displacement.

$$
\frac{\mathrm{d} \mu}{\mathrm{d} t}=\frac{1}{\tau}\left(-\mu \mathrm{e}^{-\frac{U_{1}}{k_{\mathrm{B}} T}}+(1-\mu) \mathrm{e}^{-\frac{U_{2}}{k_{\mathrm{B}} T}}\right)
$$

or, at constant deformation rate, since $\mathrm{d} \xi / \mathrm{d} t=\mathrm{d} x / \mathrm{d} t$

$$
\frac{\mathrm{d} \mu}{\mathrm{d} x}=\frac{1}{\tau \mathcal{C}}\left(-\mu \mathrm{e}^{-\frac{U_{1}}{k_{\mathrm{B}} T}}+(1-\mu) \mathrm{e}^{-\frac{U_{2}}{k_{\mathrm{B}} T}}\right)
$$

where $\tau$ is the atomic oscillation time and $c$ is the speed of deformation, $\mathrm{d} \xi / \mathrm{d} t$.

The total applied force, $P$, required to produce this deformation rate is then the integral of the local force multiplied by the jump probability over the range $-a$ to $a$

$$
P=\int_{-a}^{a}\left(\mu f_{1}(x)+(1-\mu) f_{2}(x)\right) \mathrm{d} x
$$

where $f_{1}$ and $f_{2}$ correspond to the levels of force between which the atoms jump, e.g., $Q$ and $Q^{\prime}$ at the jump location shown in Fig. 2(c).

Solution of Eq. (14) requires an expression for $U_{1}$ in terms of the displacement of the atom and for much of his analysis Prandtl assumed a simplest possible, linear dependence, i.e., $U_{1} / k_{\mathrm{B}} T=A-B x$ and $U_{2} / k_{\mathrm{B}} T=$ $A+B x$. Even with this approximation, Eq. (14) could only be solved analytically if the second, reverse terms in Eq. (14) were assumed negligible, in which case Prandtl showed that $\mu=\exp \left(-\mathrm{e}^{B X} /\left(B c \tau \mathrm{e}^{-A}\right)\right)$ and that integration of $P=\int_{0}^{a} \mu f_{1}(x) \mathrm{d} x$ gives applied force, $P$ to be

$$
P=\frac{C}{B}(\log (c)+A+\log (B \tau)-0.5772)
$$

where $C=f_{1}-f_{2}$ and was taken to be constant. This equation thus predicts that the applied force $P$ varies with the logarithm of the plastic flow rate $c$ in accord with experimental findings. Prandtl also explored a more realistic parabolic dependence of the jump energies $U_{1}$ and $U_{2}$ on displacement and discussed the dependence of plastic flow rate on temperature. Although focussed on plastic deformation of metals he showed that his model was able to predict the effective viscosity of the material under deformation 
from an essentially elastic solid at low temperature right through to viscous-like behaviour at high temperature.

Prandtl's derivation has been described in some detail above since it has been used in recent work on modelling single contact friction, described later in Section 5. However, it is clearly complex, involving a two stage integration to find the local jump probability and then to combine these at all locations to determine the total force. As such it is analytically soluble in a very limited number of cases and this may be one reason why Prandtl's model received little contemporary attention. Instead a much simpler thermalactivation model of plastic deformation developed by Richard Becker in 1925 was preferred.

\subsection{Becker's model}

Becker considered the effect of combined applied shear stress and temperature on the glide of one plane of atoms over another in a metallic crystal [6]. He defined the displacement fracture stress, $\sigma_{\mathrm{R}}$ as the stress required to produce glide in the absence of any thermal motion and the actual applied stress, $\sigma$, to be smaller than this. He then suggested that for glide to occur, thermal motion would be required within an unknown volume $V$ to bridge the difference between $\sigma$ and $\sigma_{\mathrm{R}}$, and that the mechanical energy required to achieve this would be $V\left(\sigma_{\mathrm{R}}-\sigma\right)^{2} / 2 E$ where $E$ is the Young's modulus of the material (in later work this became the elastic shear modulus $G$ ). The probability of this condition being reached is then given by

$$
p=k \mathrm{e}^{-\frac{V\left(\sigma_{\mathrm{R}}-\sigma\right)^{2}}{2 E k_{\mathrm{B}} T}}
$$

The plastic flow velocity was assumed to be proportional to this probability. Becker tested this equation using data from the deformation of tungsten single crystal monofilaments and thus determined $\sigma_{\mathrm{R}}$ and $V$. Of particular interest was the estimated value of $V$, which he found corresponded neither to the volume of a single atom nor to a whole plane but was instead about 3000 tungsten atoms. Becker thus deduced that slip originated from the concerted response to stress of a cluster of atoms within the crystal. In the 1930s, Andrade and Roscoe [26] and Becker's student Orowan $[27,28]$ combined this model with crystallographic evidence to propose that plastic deformation and creep occur due to the motion of dislocations. Becker's model was also applied in 1941 to the creep of polymers [29].

\subsection{Kauzmann}

In 1941 Kauzmann applied Eyring's model as expressed by Eq. (7) to the creep of metals [13]. This predicts that at high shear stress the underlying rate process should vary exponentially with the applied stress, rather than with the stress squared as suggested by Becker's Eq. (17). Kauzmann noted the general similarity of his and Becker's approaches however and, like Becker, found that when tested against creep data his model indicated an activation volume corresponding to several hundred atoms. He suggested that this represented the number of atoms involved in dislocation movement. Figure 3 shows Kauzmann's view of how applied stress influences the potential energy barrier for dislocation movement. It is elongated because of the multi-atom activation volume.

Kauzmann also noted that at very low stresses in the region of micro-creep, the activation volume was much smaller and close to an atomic volume, suggesting that the glide process had changed from motion of dislocations to that of single atoms.

Both Becker's and Kauzmann's models continued to be applied to describe the dependence on stress and temperature of plastic deformation, creep, and dislocation motion throughout the 1940s to 60s, with Kauzmann's model slowly displacing Becker's [30-36] (Ref. [31] is one of very few to cite Prandtl). In 1952 Cottrell [37] discussed three existing stress-augmented thermal activation models, Kauzmann's that suggested that creep varies exponentially with $\sigma$, Becker's that indicated an exponential dependence on $\sigma^{2}$ and also

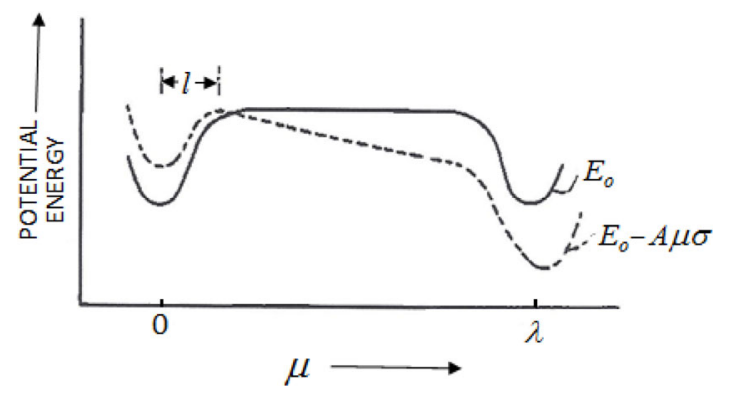

Fig. 3 Influence of applied stress on the potential energy curve of a moving dislocation. Adapted from [13]. 
a model by Mott and Nabarro that proposed an exponential $\sigma^{3 / 2}$ dependence. A detailed review of the state of the subject in the mid-1970s was provided by Kocks et al. [38].

\subsection{Since the 1970s}

The stress-augmented thermal activation concept has continued to be applied to describe the dependence of plastic flow stress on temperature and strain rate and thus the relationship between applied stress and dislocation velocity to the present day [39]. Its application has even been extended to describe the dependence of crack propagation rate on temperature and stress [40]. Models have become more complex to incorporate dislocation pinning effects and the behaviour of different crystal structures. Most recent models have replaced Becker's original volume $V$ by the product of an activation area and the Burger's vector (an activation length) and have allowed this activation area to vary with the applied stress [41].

Although most interest has been in the plastic deformation of metals, both Becker's [29] and Eyring's model $[42,43]$ have also been applied to the deformation of solid polymers. In 1971 Ward analysed tensile and compressive yield stress data of several polymers as a function of temperature and strain rate in terms of an extended Eyring model [43]

$$
\dot{\varepsilon}=A \mathrm{e}^{-\frac{(\Delta U-\tau v+p \Omega)}{k_{\mathrm{B}} T}}
$$

where $\dot{\varepsilon}$ is the strain rate, $\Delta U$ an activation energy for the flow process, $\tau$ the shear yield stress, $v$ the shear activation volume, $p$ the hydrostatic pressure, and $\Omega$ the pressure activation volume. Ward thus introduced a pressure work term in the rate equation which he assumed was positive, i.e., the rate decreased with increasing pressure. This dependence on pressure had been previously used by Eyring et al. to describe the influence of pressure on viscosity [44] and, as will be described in Section 5 of this paper, was later adopted by Tabor to describe the influence of load on sliding friction. In many ways a pressure term is more familiar to thermodynamicists than a shear stress term, since a PV work term is embedded in rate and equilibrium equations involving gases.

\section{Lubricant viscosity and rheology}

\subsection{Eyring's model}

In 1936 Eyring developed his well-known model of liquid viscosity. This originated from his transition state theory of chemical kinetics that considers a chemical reaction as involving passage of a molecular system over an energy barrier in the process of converting reactants to products. Eyring thus considered liquid flow as resulting from passage of individual molecules over an energy barrier and, as the title of his paper indicates, regarded viscosity as an example of a reaction rate. Figure 4 shows his schematic model in which a molecule moves across an adjacent plane of molecules into a vacancy or "hole" under the application of a shear force $f$ [14]. To do this it must traverse an activation energy barrier $E_{\mathrm{a}}$ as shown earlier in Fig. 1 . The applied force on the molecule has the effect of lowering this activation energy by a factor $f \lambda / 2$ in the direction of the force and raising it by this amount in the backward direction. This causes the molecule (and the other molecules in its plane) to move relative to the adjacent plane at velocity given by Eq. (8), i.e., $\Delta u=2 k_{\mathrm{o}} \lambda \sin h\left(f \lambda / 2 k_{\mathrm{B}} T\right)$. The velocity gradient or shear rate is thus

$$
\dot{\gamma}=\frac{\Delta u}{\lambda_{1}}=\frac{2 k_{\mathrm{o}} \lambda}{\lambda_{1}} \sin h\left(\frac{f \lambda}{2 k_{\mathrm{B}} T}\right)
$$

where $k_{\mathrm{o}}$ is the rate constant in the absence of an applied force, $\lambda_{1}$ is the spacing between adjacent molecular layers and $\lambda$ is the total distance the molecule moves in one jump, i.e., $2 \Delta x$ (the distance between the two energy minima in Fig. 1).

The force on a molecule can be related to the applied shear stress by $\tau=f / \lambda_{2} \lambda_{3}$, where the product

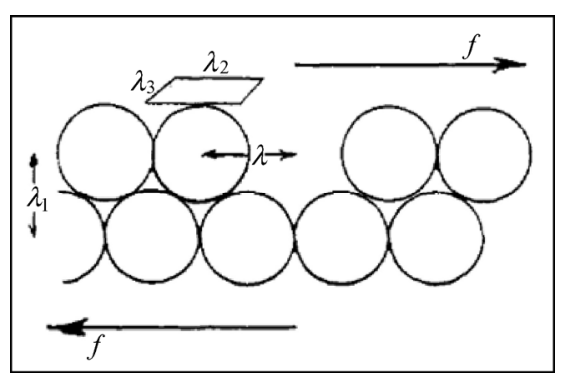

Fig. 4 Eyring's schematic model of viscous flow of simple molecules. Reproduced with permission from [14]. Copyright ACS, 1940. 
$\lambda_{2} \lambda_{3}$ is the cross sectional area of the molecule in the plane of shear as shown in Fig. 4.

Replacing $f$ in Eq. (19) by the shear stress $\tau$ yields an expression for the effective viscosity

$$
\eta_{\text {eff }}=\frac{\tau}{\dot{\gamma}}=\frac{\tau \lambda_{1}}{2 k_{\mathrm{o}} \lambda \sin h\left(\frac{\tau \lambda_{2} \lambda_{3} \lambda}{2 k_{\mathrm{B}} T}\right)}
$$

At low applied shear stress, when $\tau \ll<k_{\mathrm{B}} T / \lambda_{2} \lambda_{3} \lambda$, $\sin h\left(\tau \lambda_{1} \lambda_{2} \lambda / 2 k_{\mathrm{B}} T\right)=\tau \lambda_{1} \lambda_{2} \lambda / 2 k_{\mathrm{B}} T$, so the Newtonian viscosity is given by

$$
\eta_{\mathrm{N}}=\frac{k_{\mathrm{B}} T \lambda_{1}}{k_{\mathrm{o}} \lambda_{2} \lambda_{3} \lambda^{2}}
$$

Eyring used this expression in two ways. Firstly, he evaluated the rate constant $k_{\mathrm{o}}$ by relating the energy of activation $E_{\mathrm{a}}$ to the enthalpy of vaporisation and including a free volume term [8]. Eyring found that that for liquids with approximately spherical molecules Eq. (21) predicted the measured Newtonian viscosity quite well, based on $\lambda^{2} \lambda_{2} \lambda_{3} / \lambda_{1}$ being the molecular volume. For polymeric molecules however, the predicted viscosity was too low and Eyring suggested that this was because in the latter case the flow unit was only a segment of the molecule [14].

Eyring also used Eq. (21) to substitute $k_{\mathrm{o}}$ in Eq. (20) and so obtained a molecular-based model of shear thinning behaviour of liquids

$$
\frac{\eta_{\text {eff }}}{\eta_{\mathrm{N}}}=\frac{\tau \lambda_{2} \lambda_{3} \lambda}{2 k_{\mathrm{B}} T \sinh \left(\frac{\tau \lambda_{2} \lambda_{3} \lambda}{2 k_{\mathrm{B}} T}\right)}
$$

The term $2 k_{\mathrm{B}} T / \lambda_{2} \lambda_{3} \lambda$ has units of stress and is nowadays called the Eyring stress, $\tau_{\mathrm{e}}$. We thus have

$$
\frac{\eta_{\text {eff }}}{\eta_{\mathrm{N}}}=\frac{\tau}{\tau_{\mathrm{e}} \sin h\left(\frac{\tau}{\tau_{\mathrm{e}}}\right)}
$$

Or, since $\eta_{\text {eff }}=\tau / \dot{\gamma}$

$$
\tau=\tau_{\mathrm{e}} \sin h^{-1}\left(\frac{\eta_{\mathrm{N}} \dot{\gamma}}{\tau_{\mathrm{e}}}\right)
$$

This remarkably simple expression was the first theoretically-derived rheological model of liquid shear thinning. It originates directly from the stressaugmented thermal activation concept and its relationship to this can be most clearly seen by the rearrangement of Eq. (24) to

$$
\frac{\eta_{N} \dot{\gamma}}{\tau_{\mathrm{e}}}=\sin h\left(\frac{\tau}{\tau_{\mathrm{e}}}\right)=\frac{\mathrm{e}^{\tau / \tau_{\mathrm{e}}}-\mathrm{e}^{-\tau / \tau_{\mathrm{e}}}}{2}
$$

This has obvious analogies to Eq. (7), showing how the net flow rate is a combination of flow in the forward and backward directions and how these are separately influenced by the applied force and thus shear stress. It also shows that this $\sin h$ relationship possesses the requisite symmetry properties for a rheology equation in that, when the sign of the stress is changed, this results in the shear rate changing sign but not magnitude.

Equation (24) predicts that simple molecular liquids such as most base oils will begin to show significant shear thinning when the value of $\eta_{\mathrm{N}} \dot{\gamma} / \tau_{\mathrm{e}}$ approaches unity. However we now know that for such liquids, $\tau_{\mathrm{e}}$ is typically 5 to $50 \mathrm{MPa}$ and it was not possible in Eyring's day to reach such values of the product of $\eta_{\mathrm{N}}$ and $\dot{\gamma}$ without excessive shear heating. Instead Eyring's shear thinning model was applied, arguably less appropriately, to more complex fluids such as polymer melts and solutions and colloidal dispersions that shear-thinned much more readily [45]. Eyring's original model assumes one type of flow unit and in 1955 Ree and Eyring [46] extended the model to accommodate blends of molecules with very different size flow units so that

$$
\tau=\sum_{i=1}^{n} \frac{x_{i}}{\alpha_{i}} \sin h^{-1}\left(\beta_{i} \dot{\gamma}\right)
$$

where $n$ is the number of different flow units present, $x_{i}$ is the fractional area of a plane parallel to the shear stress occupied by the $i$-th flow unit and $\alpha_{i}$ and $\beta_{i}$ are defined as $\alpha_{i}=\left(\lambda \lambda_{2} \lambda_{3}\right)_{i} / 2 k_{\mathrm{B}} T$ and $\beta_{i}=1 /\left(2 k_{\mathrm{o}} \lambda / \lambda_{1}\right)_{i}$. $\alpha$ is effectively the reciprocal of the Eyring stress for that component. Unfortunately, the complexity of this approach detracts from the simple elegance of the original model and its application has been limited. It has been used primarily to describe the flow of polymer solutions [47] or colloidal dispersions [48] with just two 
components although one three component system has been examined [49].

\subsection{EHD friction}

In the 1960s there was growing interest in the friction of high pressure elastohydrodynamic (EHD) lubricated contacts, as found in gears, cams, and rolling bearings. This friction was much lower than calculated by assuming that the lubricant behaved in a Newtonian fashion, indicating that the combination of the high strain rate and very high pressure (and thus very high viscosity) present in such contacts was resulting in shear thinning, even of quite low molecular weight lubricants. Initially this was ascribed to the existence of a limiting shear stress for the liquid [50,51] although Plint [51] also noted a dependence of effective viscosity on $\log$ (shear rate) in low slip conditions. Then, in the 1970s, Hirst and Moore [52-55] and Johnson and Tevaarwerk $[56,57]$ showed that disc machine friction measurements could be very well explained by Eyring's shear thinning model as expressed in Eq. (24). In such measurements, and indeed all measurements at a combination of high viscosity and high shear rate, it is important to eliminate thermal effects and this was done initially by studying very low sliding speed conditions and later by applying an isothermal correction $[58,59]$.

Figure 5 shows plots of isothermal friction coefficient versus shear rate from Evans and Johnson for a series of disc machine tests on a mineral oil at varies applied loads and thus mean contact pressures [59]. The mean shear stress is simply the product of the friction coefficient and the mean pressure so these plots are equivalent to shear stress versus shear rate.

The results were fitted to

$$
\mu=\bar{p} \tau_{\mathrm{e}} \sin h^{-1}\left(\frac{\eta_{N} \dot{\gamma}}{\tau_{\mathrm{e}}}-\frac{\eta_{N}}{\tau_{\mathrm{e}} G} \frac{\mathrm{d} \tau}{\mathrm{d} t}\right)
$$

where $\mu$ is the friction coefficient, $\bar{p}$ the mean pressure, and $G$ the combined shear modulus of the surfaces and lubricant. The first term in the brackets is clearly the Eyring viscous response, while the second term represents the reduction in friction due to elastic compliance of the surfaces and the lubricant film in response to the rapidly increasing shear

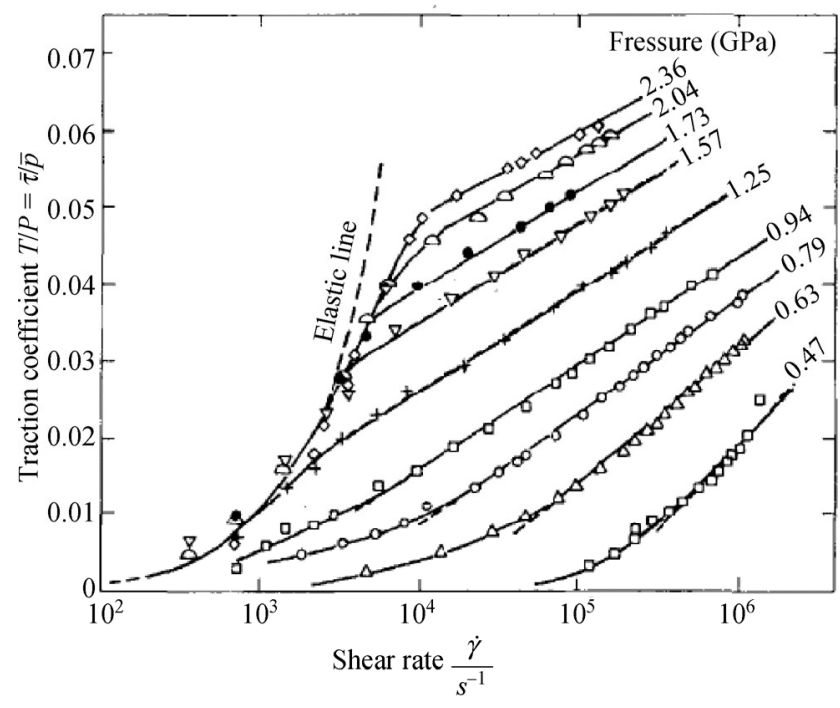

Fig. 5 Isothermal friction coefficient versus shear rate for a hydrocarbon lubricant in an EHD contact. Reproduced with permission from [59]. Copyright I.Mech.E., 1986.

stress in the inlet half of the contact. This elastic term becomes negligible at high shear rate.

At low pressures and high shear rates the curves follow closely Eyring's $\sin h^{-1}$ relationship between shear stress and shear rate. At high pressures and shear rates these curves appear as straight lines, indicating that shear stress is proportional to $\log$ (shear rate). This is fully consistent with Eq. (24) since, when $\tau>\tau_{\mathrm{e}}$, the equation approaches $\tau=\tau_{\mathrm{e}} \log _{\mathrm{e}}\left(2 \eta_{\mathrm{N}} \dot{\gamma} / \tau_{\mathrm{e}}\right)$.

Many other experimental studies have confirmed the above findings and the Eyring shear thinning equation has become widely used for modelling the behaviour of liquid lubricants in EHD conditions [60-66]. It has also been extended and applied to glass-forming liquids [67]. It is not without controversy and some researchers have advocated alternative shear thinning equations that were originally developed to study polymer shear thinning [45]. However, polymer shear thinning occurs at relatively low shear stress, while the phenomenon of simple, low molecular weight liquids exhibiting extensive shear thinning is practically unique to tribology. This is because only in EHD lubricated contacts are liquids routinely subjected to the extreme pressure and shear rate conditions required to produce shear stresses significantly in excess of the Eyring stress.

A recent development is the application of nonequilibrium molecular dynamics simulation (NEMD) 
to model the molecular behaviour of large ensembles of liquid molecules under shear. Such simulations confirm the logarithmic dependence of shear stress on shear rate predicted by Eyring at high shear stress for most of the liquids studied and also, in some cases, the whole $\sin h^{-1}$ transition from linear Newtonian dependence at low shear rate to logarithmic dependence at high shear rate [68-70]. Figure 6 shows the predicted stress-strain rate behaviour of squalane, which matches the Eyring model over an extremely wide shear rate range [70]. This is not definite confirmation of the validity of the Eyring model however since other models show equally good fit within the limits of accuracy of the simulations [71, 72]. Interestingly some liquids do not follow this type of Eyring dependence, instead showing a levelling out of shear stress with increasing strain rate to reach a limiting shear stress [69].

Finally, it should be noted that, while all of the above has focussed on bulk rheology, Eyring's model has also been applied to describe the shear rheology

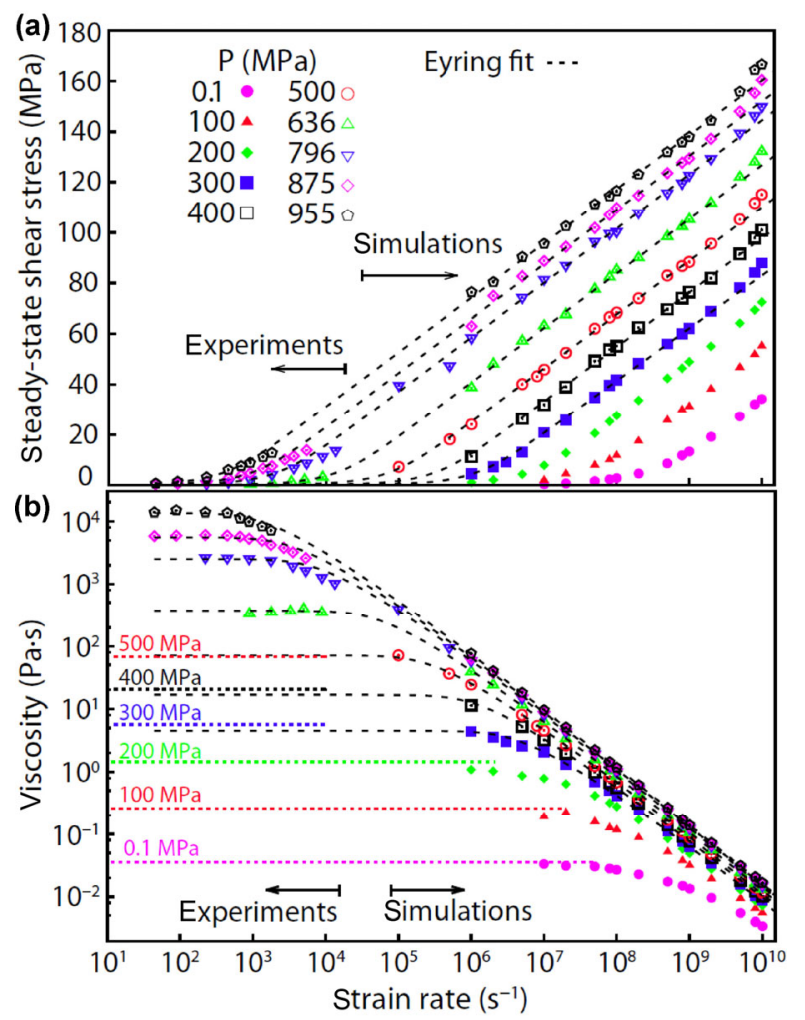

Fig. 6 NEMD simulations of squalane showing the dependence of predicted shear stress and viscosity on strain rate and temperature (dashed lines are Eyring fits). Reproduced with permission from [70]. Copyright NAS, 2017. of very thin adsorbed films $[12,73]$. This is important in terms of the impact of surfactant and polymers on the stability of foam and emulsions but may also have relevance to the rheology of nanoscale, confined films.

\section{Sliding friction}

\subsection{Polymer friction}

Although both Prandtl and Eyring noted the applicability of their stress-augmented thermal activated models to kinetic friction, it was only in the 1950s that Schallamach [17] and Bartenev [74] employed it in this context, to explain the speed, load, and temperature dependence of the adhesive sliding friction of polymers and elastomers. Initially both researchers envisaged polymer friction as resulting from the interlocking of the molecular force fields between rubber and track at the real area of contact, a concept very similar to Eyring's viscous flow. However subsequent work indicated that at very high sliding speeds friction started to decrease, as shown in Fig. 7 [75]. To explain this, Schallamach's interpretation shifted towards sliding involving the breaking and reforming of bonds, with bond-forming being a thermally activated process but bond-breaking involving stress-augmented thermal activation [76]. He also

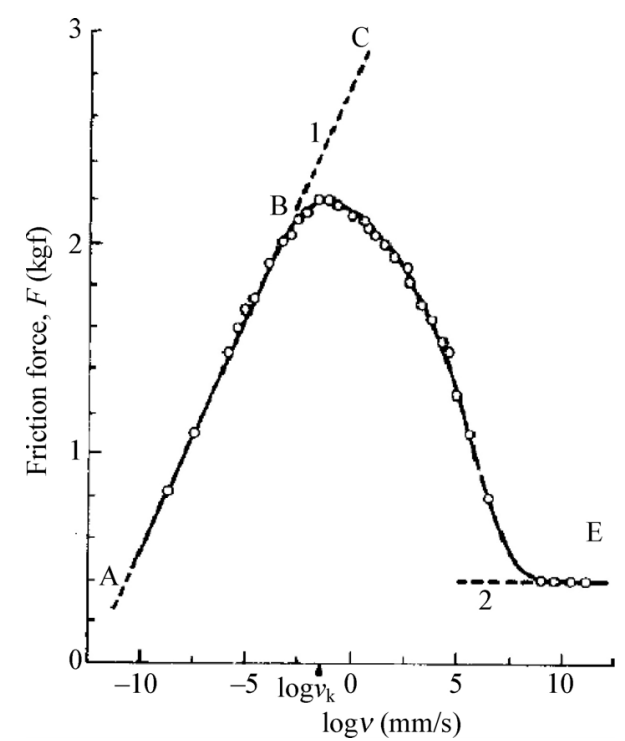

Fig. 7 Friction versus $\log$ (sliding speed) for a vulcanised styrenebutadiene copolymer rubbed against polished steel at $40{ }^{\circ} \mathrm{C}$ and $0.65 \mathrm{~kg}$ normal load. Reproduced with permission from [75]. Copyright Elsevier, 1965. 
considered the bonds to be elastic so that the forces they experienced were proportional to how far they were stretched. The effect of these two assumptions was to introduce a maximum in the friction versus speed curve since friction depended on factors that both increased with sliding speed (bond stretching and thus force), and reduced with sliding speed (lifetime and thus number of bonds).

Bartenev's explanation for the drop in friction differed from Shallamach's. He found that the fall in friction at high sliding speeds corresponded to a similar fall as temperature was reduced. He thus invoked time-temperature transformation to suggest that at high sliding speeds, the polymer's mechanical properties close to the interface changed to become similar to its properties at its glass transition temperature [75].

A considerable problem in developing models of polymer friction lies in fact that the real area of contact can vary considerably with speed and load due to surface deformation and junction growth and this change in contact area can obscure intrinsic variations of interfacial shear strength with load and speed. To address this issue, both Schallamach and Bartenev carried out studies to observe the real area of contact during the sliding of elastomers. Bartenev et al. measured real contact area and friction simultaneously while increasing sliding speed and his findings supported the logarithmic dependence of friction on speed predicted by his model [77]. Schallamach, by contrast, observed the presence and motion of deformation waves now termed Schallamach waves for polymers with low elastic modulus [78].

Schallamach's initial model of polymer friction was applied by Stejin in 1968 to interpret the measured friction properties of PTFE [79], while his bond-breaking and reforming model was considerably refined in 1986 by Chernyak and Leonov to take account of the actual force-extension properties of polymer chains and the way that momentum is transferred from polymer to surface during this stretching process [80].

In the late 1970s Tabor and co-workers studied the dependence of the friction properties of polymers on load, sliding, and temperature [81, 82]. By using thin polymer films of thickness $c a 200 \mathrm{~nm}$ on very smooth, rigid substrates, contact areas close to those predicted by Hertz were obtained so that mean shear stress could be determined from the measured friction. They found that shear stress was proportional to pressure and increased linearly with $\log$ (sliding speed) at a fixed temperature for most polymers, although one polymer, PMMA, gave shear stress that reduced linearly with speed at low temperature. This anomalous result was ascribed to a viscoelastic response [82]. They developed an empirical equation relating stress to pressure, sliding speed, and temperature closely based on stress-augmented thermal activation.

Much more recently Bouhacina et al. have used an AFM to study the friction properties of polymer films grafted on a silicon substrate [83]. Like Tabor, they found friction to increase with $\log$ (sliding velocity) and interpreted this in terms of Eyring's model.

\subsection{Boundary friction}

In 1982 Briscoe and Evans studied the influence of load, sliding speed, and temperature on the boundary friction of sliding surfactant monolayers [84]. The test set-up involved a pair of crossed, mica-covered glass prisms and monolayers of fatty acids and fatty acid soaps were deposited on the mica surfaces using the Langmuir Blodgett method. Optical interferometry was used to measure the separation of the mica surfaces and also the precise contact area during sliding. This approach gave very accurate measurement of shear stress of the sliding contact.

For fatty acid monolayers, shear stress, $\tau$, was found to increase linearly with load, to increase with $\log$ (sliding speed) (Fig. 8(a)) and to decrease linearly with temperature (Fig. 8(b)). Similar behaviour was also seen when multilayers were used. With fatty acid soaps formed by depositing fatty acids at high $\mathrm{pH}$, friction was constant or even fell with sliding speed.

Briscoe and Evans interpreted their fatty acid results in terms of the Eyring model with an added pressure work term as introduced by Ward [43], to give the equation for the average velocity of a molecule at the sliding interface

$$
v=2 v b \mathrm{e}^{-\left(\frac{Q^{\prime}+\Omega p}{k_{\mathrm{B}} T}\right)} \sin h\left(\tau \varphi / k_{\mathrm{B}} T\right)
$$

where $v$ is the effective vibration frequency of the sliding molecules, $b$ is the distance across the energy barrier, $Q^{\prime}$ is the thermal activation energy for the flow process, $\Omega$ is the pressure activation volume, 

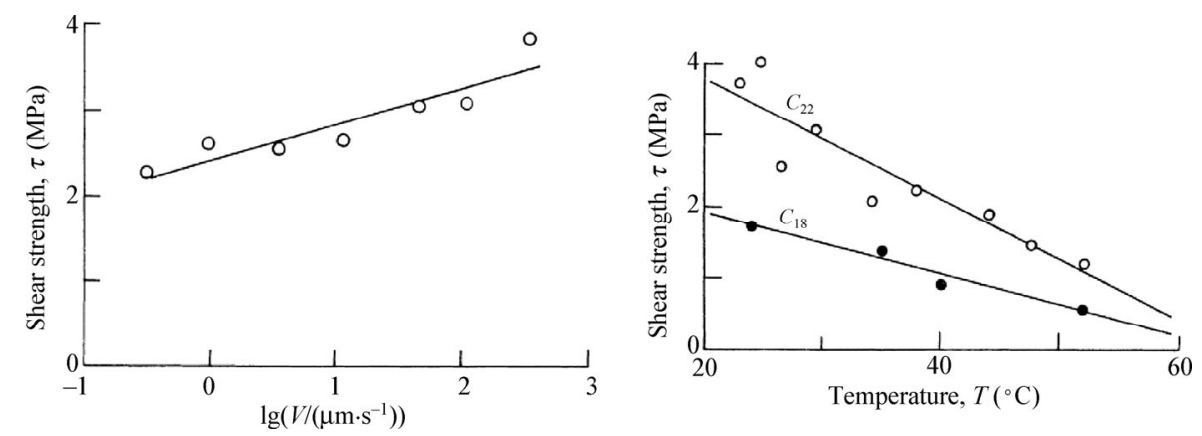

Fig. 8 (a) Shear strength of stearic acid monolayers at $21^{\circ} \mathrm{C}$. (b) Shear strength against temperature for stearic and behenic acid monolayers. Reproduced with permission from [84]. Copyright The Royal Society, 1982.

and $\phi$ is the shear activation volume. Assuming the sliding velocity, $V$ is proportional to the average molecular velocity gives

$$
V=2 V_{\mathrm{o}} \mathrm{e}^{-\left(\frac{Q^{\prime}+\Omega p}{k_{\mathrm{B}} T}\right)} \sin h\left(\tau \varphi / k_{\mathrm{B}} T\right)
$$

where $V_{\mathrm{o}}$ is an unknown velocity constant.

At high stresses this becomes

$$
V=V_{\mathrm{o}} \mathrm{e}^{-\left(\frac{Q^{\prime}+\Omega p-\tau \varphi}{k_{\mathrm{B}} T}\right)}
$$

or

$$
\tau=\frac{k_{\mathrm{B}} T}{\varphi} \log \left(\frac{V}{V_{\mathrm{o}}}\right)+\frac{1}{\varphi}\left(Q^{\prime}+p \Omega\right)
$$

This predicts the linear dependence of $\tau$ on $p$ and $T$ and its logarithmic dependence on $V$ found experimentally. Based on this equation, at constant sliding speed and temperature it also predicts the two term Derjaguin equation for friction where

$$
F=c+\alpha W
$$

and the constant $\alpha$ corresponds to $\Omega / \phi$. Based on estimated values of the shear activation volume, $\phi$, Briscoe and Evans concluded that a flow unit comprised several fatty acid molecules. They interpreted the pressure activation volume, $\Omega$, as the local increase in volume needed to permit molecular motion and found that this corresponded to about the volume of an ethyl group.

Subsequently a number of studies have studied the friction of surfactant solutions in hydrocarbons in macro-scale sliding contacts and found that friction depended on log(sliding speed), in accord with Briscoe and Evans' model [85-87]. This behaviour appears to depend strongly on the ability of the surfactant to form a close-packed monolayer, so that if this layer is disrupted in some fashion, friction tends to become independent of speed. Thus Ingram et al. found that some blends of low and high chain length fatty acids gave speed-independent friction even though solutions of the individual acids gave logarithmic dependence [86]. Campen et al. found that while oleic acid solutions gave friction constant with speed, the same concentration of its trans-isomer, elaidic acid, showed logarithmic dependence, similar to stearic acid [87]. The trans-isomer is able to pack more closely in absorbed films than the cis-oleic acid.

In 2003, Drummond et al. used a surface forces apparatus (SFA) to measure the friction properties of a pair of cationic surfactant-coated mica surfaces in aqueous solution over a wide sliding speed range [88]. They were able to measure the separation and the precise contact area using optical interferometry and thus convert friction to shear stress. Four stages of behaviour were observed, as shown in Fig. 9. At very low sliding speeds shear stress increased with $\log$ (sliding speed), before levelling out to a constant value. At still higher speed stick slip was observed with the friction and thus shear stress transiting between high and low values. For some surfactant concentrations at very high sliding speeds, smooth sliding returned, with shear stress again increased approximately with $\log$ (speed).

Drummond and coworkers interpreted these results in terms a stress activation model based closely on Shallamach's 1963 model [76], with the contact consisting of many nano-junctions that can form, stretch, 


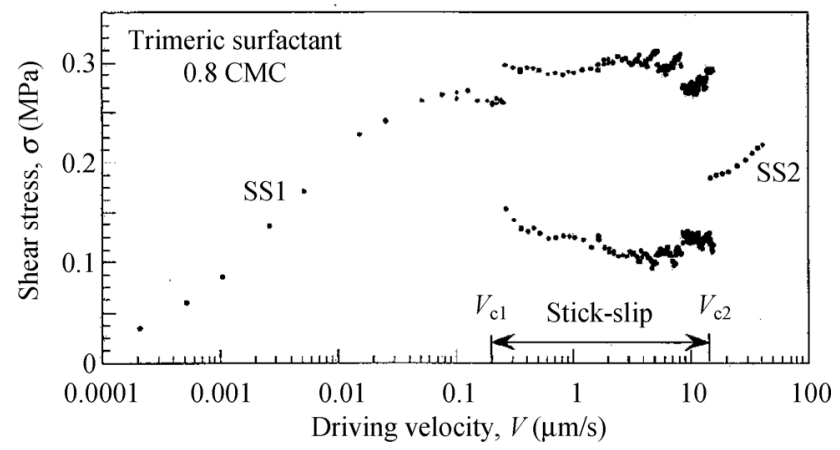

Fig. 9 Dependence of shear stress on driving velocity while shearing two adsorbed monolayers of trimeric ammonium tribromidebased surfactant from aqueous solutions at $T=20{ }^{\circ} \mathrm{C}$. Reproduced with permission from [88]. Copyright AIP, 2003.

and break independently. Thus the first stage of the shear stress/speed curve is dominated by the Eyring principle of stress-augmented thermally activated breaking of these junctions with the now recognisable $\sin h^{-1}$ or, at higher speed, logarithmic dependence of shear stress on sliding speed. Eventually this process saturates and the shear stress levels out before starting to fall as the number of nano-junctions reduces. The stick slip stage ensues as a result of the well-known instability that occurs in elastic systems when static friction is lower than dynamic friction. Eventually, at very high speeds Drummond et al. suggested that all nano-junctions were broken and friction originated from thin film viscous shear. Mazuyer et al. have recently applied Drummond's model to the measured friction behaviour of solutions of two organic friction modifiers in polyalphaolefin base fluid in a surface forces apparatus [89].

\subsection{AFM friction}

In the late 1980s atomic force microscopes (AFMs) started to be used to study friction. Initial work focussed on the stick-slip behaviour generally observed in this system and this was analysed in terms of the PrandtlTomlinson model [90]. However, in many cases, both mean and maximum friction also increased with $\log$ (speed) and in 1990 this was analysed by Gnecco et al. [91] using a stress-augmented thermal activation model very similar to Prandtl's 1928 concept [2]. They considered an AFM tip, elastically constrained by a cantilever, sliding up the flank of a sinusoidal force field in a system identical to that shown in Fig. 2. They described the change in probability of it not having jumped (i.e., the tip not having slipped forward), $p(t)$ with time $t$ as

$$
\frac{\mathrm{d} p(t)}{\mathrm{d} t}=-f_{\mathrm{o}} \mathrm{e}^{-\Delta E^{+}(t) / k_{\mathrm{B}} T} p(t)
$$

where $f_{\mathrm{o}}$ is a characteristic vibration frequency and $\Delta E^{+}$is the energy needed to overcome the remaining potential barrier in the forward direction (equivalent respectively to the reciprocal of the oscillation time $\tau$ and to $U_{1}$ in Prandtl's Eq. (14)). This equation is couched in terms of probability of not having jumped since the jump rate at time $t$ will be proportional to this.

In principle, the product of this equation and the corresponding elastic force at time $t$ could be integrated to relate the total force to the velocity, as was done by Prandtl, but instead Gnecco et al. transformed it in terms of the force $F$ using $\mathrm{d} p(t) / \mathrm{d} t=$ $(\mathrm{d} p(F) / \mathrm{d} F) \cdot(\mathrm{d} F / \mathrm{d} t)$, so that

$$
\frac{\mathrm{d} p(F)}{\mathrm{d} F}=-f_{\mathrm{o}} \mathrm{e}^{-\Delta E^{ \pm}(F) / k_{\mathrm{B}} T} p(F)\left(\frac{\mathrm{d} F}{\mathrm{~d} t}\right)^{-1}
$$

They then expressed this in terms of sliding velocity, $v$ using $\mathrm{d} F / \mathrm{d} t=(\mathrm{d} F / \mathrm{d} x) \cdot(\mathrm{d} x / \mathrm{d} t)$ to give

$$
\frac{\mathrm{d} p(F)}{\mathrm{d} F}=-f_{\mathrm{o}} \mathrm{e}^{-\Delta E^{+}(F) / k_{\mathrm{B}} T} p(F) k_{\text {eff }} v
$$

where $k_{\text {eff }}$ is the effective spring constant of the cantilever.

They assumed a linear reduction of energy with force

$$
\Delta E^{+}(F)=\lambda\left(F^{*}-F\right)
$$

where $\lambda$ is effectively an activation length (the distance from the bottom to the top of the eenrgy barrier) and $\lambda F^{*}$ is the energy needed for the tip to jump at absolute zero temperature (the thermal activation energy needed to jump in the absence of an applied force).

To solve Eq. (35), Gnecco et al. assumed that jump would occur at the maximum probability position where $\mathrm{d}^{2} p(F) / \mathrm{d} F^{2}=0$ and so obtained the force at the most probably position for the AFM tip to slip

$$
F(v)=F^{*}+\frac{k_{\mathrm{B}} T}{\lambda} \log _{\mathrm{e}}\left(\frac{v k_{\mathrm{eff}} \lambda}{f_{\mathrm{o}} k_{\mathrm{B}} T}\right)
$$

From the above, the close analogy between this approach and Prandtl's should be evident, with 
Prandtl's single elastically-constrained atom being substituted by a single elastically-constrained AFM tip. It does not, however, appear that Gnecco et al. were aware of Prandtl's previous work and it was not until about 2002 that Prandtl's 1928 paper started to be referenced in this context [92].

In subsequent research, Gnecco's approach was extended to include a more realistic, parabolic dependence of energy on force, i.e., $\Delta E^{+}(F) \propto\left(F^{*}-F\right)^{3 / 2}$ and this predicted a dependence of friction force on $\log (v)^{2 / 3}$ rather that a $\log (v)$ [93]. Unfortunately it is quite different to test the validity of this without measuring the variation of friction with speed over a speed range of at least two orders of magnitude.

\subsection{Friction simulation}

As well as the above analytical approach, there have been a considerable number of studies applying molecular dynamics simulation (MDS) to model sliding friction of tethered or adsorbed monolayer boundary films [94-100]. The majority of these have found friction to increase with $\log$ (sliding speed) and compared this with Briscoe and Evans' stressaugmented thermal activation equations. However, some simulations have predicted other types of friction/ speed response. Chandross et al. found friction to be proportional to $\log$ (sliding speed) at low loads but to be independent of speed at high loads [96]. Chen et al. found that monolayers films, and indeed bare substrates, that terminated with hydrogen acceptor/ donor species and could thus form links across the contact gave friction that decreased with speed, while those that could not form such cross links showed friction proportional to $\log$ (sliding speed) [98].

In almost all of the above friction studies, both experimental and modelling, the main finding suggestive of stress-augmented thermal activation has been that the friction force or shear stress increases proportional to $\log$ (sliding speed). According to both Prandtl's and Eyring's models this should, of course, only be the case at high stresses when the backward jump process becomes negligible. As will be discussed later in this paper this is generally true in the context of sliding friction where contact pressures and thus shear stresses are likely to be very high. One exception is Drummond's study where at very low sliding speeds an initial linear dependence of friction on speed was observed. However this study used a surface forces apparatus where contact pressures are generally much lower than in normal rough surface tribological contacts.

In 2011 Müser used molecular dynamics simulation to predict dry friction over an extremely wide sliding velocity range and found friction to vary with $\sin h^{-1}$ (velocity) as shown in Fig. 10 [101]. Although Müser did not interpret this explicitly in the context of combined forward and reverse transitions, the similarity to the prediction of the Eyring's model is quite striking.

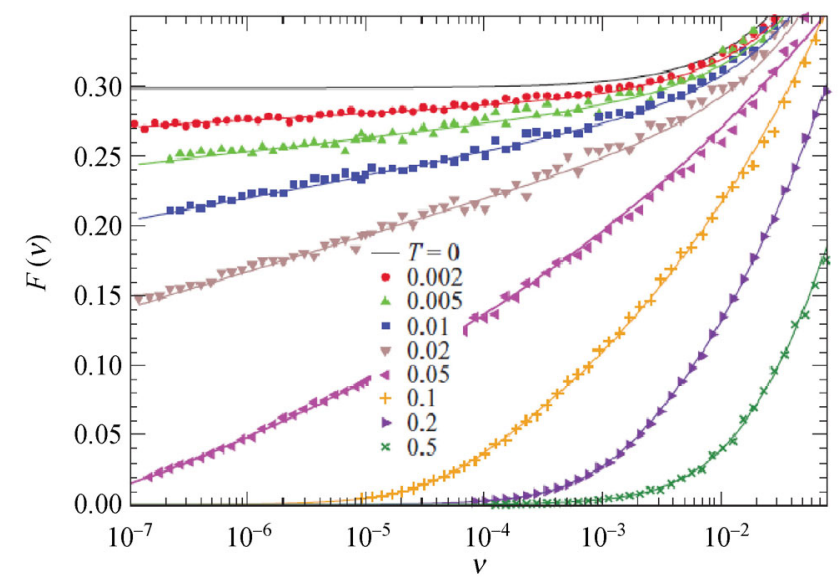

Fig. 10 Variation of kinetic friction with sliding velocity and temperature predicted from a molecular dynamics model. Reproduced with permission from [101]. Copyright AIP, 2011.

\section{Mechanochemistry}

\subsection{Background}

A fourth area of application of stress-augmented thermal activation to Tribology concerns mechanochemistry and this section will first briefly consider mechanochemistry as applied to chemistry as a whole and then focus on its specific application to Tribology.

The idea that mechanical forces can promote chemical reactions has a long history. Thus, while studying the reaction of silver salts with reactive metals, Faraday in 1820 noted [102]:

"If dry chloride of silver in powder be triturated in a mortar with zinc filings, then the two bodies immediately act, and a heat above that of boiling water is produced."

The actual term mechanochemistry appears to have been coined by Ostwald in 1909 although the 
mechanical force that he had in mind was pressure rather than shear [103].

As described in Section 2 of this paper, the concept that mechanochemistry might originate from a combination of stress and thermal activation causing chemical bonds to break was first developed by Beuche and Zhurkov in the 1950s, both of whom developed reaction rate models that added a work energy term to the Arrhenius equation (Eqs. (11) and (12), respectively). Figure 11 shows some of Zhurkov's data relating the lifetime to fracture, $\tau$ (the reciprocal of the fracture rate), to the applied tensile stress for a metal, a ceramic, and an organic glass, illustrating both the exponential dependence of lifetime on tensile stress and also the impact of temperature on reducing the fracture lifetime [24].

For metals and ceramics, Zhurkov suggested that his thermal activation energy $\left(U_{\mathrm{o}}\right.$ in Eq. (12)) represented the binding energy of metallic and ionic bonds. For polymers it was not clear whether covalent bonds within molecules were being broken or van der Waal bonds between polymer molecules, allowing the latter to slip past one another. However, in 1974 Zhurkov and Kursukov used infrared spectroscopy to monitor the breaking of covalent polymer bonds and showed that this process was paramount in determining the dependence of fracture on stress [25]. They also showed that the values of $U_{\mathrm{o}}$ calculated from the dependence

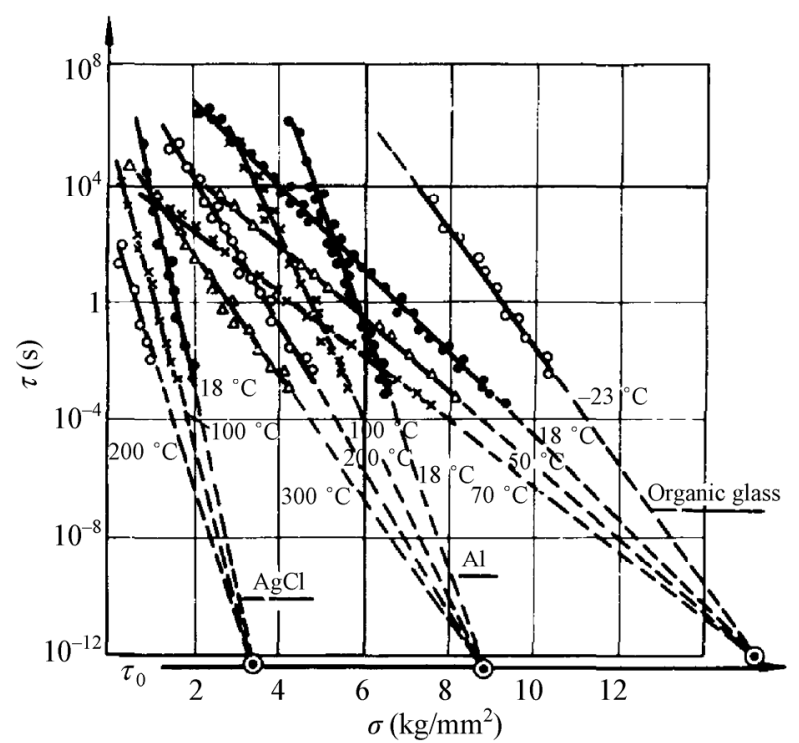

Fig. 11 Measured lifetime to fracture versus applied tensile stress for three materials at various temperatures. Reproduced with permission from [24]. Copyright Springer, 1965. of fracture time on temperature in Eq. (12) were the same as those in thermal degradation experiments for the polymers tested, writing:

"The equality of the activation energies indicates that a connection exists between mechanical, mechanochemical, and thermal destruction of polymers [25]."

Interestingly this appears to be the first time Zhurkov employs the term "mechanochemical" in one of his papers.

In the field of chemistry an important stage in the development of mechanochemistry was made by Bell in 1978 [104]. He applied Zhurkov's model to describe the cross-linking reactions of biomolecules such as proteins with cell membranes and the consequent effect of this on cell-cell and cell-surface adhesion. He showed that the kinetics of this process was welldescribed if the debonding process was driven by Zhurkov's stress-augmented thermal activation lifetime equation (Eq. (12)).

In the last two decades mechanochemistry has become a defined area of research in the field of chemistry and its significance can be gauged from the considerable number of review papers on the subject in recent years [105-110]. Bell's 1978 paper is generally taken as the seminal starting point, although occasionally earlier work by Zhurkov and Eyring is cited. Mechanochemistry is now being applied to a wide range of areas of chemistry and biochemistry, including solvent free synthesis routes [108, 111], molecular motors $[112,113]$, single molecule studies, for example using molecular tweezers or AFMs [114, 115], cell differentiation [116, 117], and mechanophores [118, 119]. The latter are molecular groups designed specifically to respond to mechanical force, to enable this force to be measured externally (sensors) or to produce materials that respond in desired ways to applied forces (smart materials).

Before discussing the application of mechanochemisty to Tribology it is important to define what is meant by this term in the context of the current review. The IUPAC gives a very broad definition of a mechano-chemical reaction being a "chemical reaction that is induced by the direct absorption of mechanical energy" [108]. This has led to some confusion about whether the term "mechanochemistry" should encompass indirect chemical effects of mechanical force 
on solids, such as local temperature rise or increase of surface area [108]. Within tribology such a broad definition would, of course, encompass all of tribochemistry, and nowadays most chemists and also most tribologists limit the term mechanochemistry specifically to describe chemical processes where the application of a mechanical force to individual molecules directly promotes bond breaking and thus increases chemical reaction rate. The reaction rate will then depend on applied force and temperature according to a stress-augmented thermal activation rate equation. This focussed definition will be the one assumed in the current review.

\subsection{Mechanochemistry in Tribology: Polymer degradation}

Until very recently the only application of stress augmented thermal activation to chemical reactions in Tribology concerned polymeric viscosity modifier additives. Polymers are especially susceptible to damage by mechanical forces since these forces acting on many monomers in the chain can accumulate to produce large tensile stresses at mid chain or entanglement points and so rupture the chain. This effect became an important bulk polymer processing tool in the 1920s and was patented in 1937 for use in producing viscosity modifiers additives [120]. Then, in the 1940s it was found that very high molecular weight (MW) polymers in solution could show such chain rupture when subjected to high shear rates, resulting in permanent viscosity loss and limiting the molecular weight of polymers that could be used in lubricants [121]. A short review of permanent viscosity loss of viscosity modifiers has recently been provided by Marx et al. [122], while a general review of the research on the mechanochemistry of polymers up to the late 1980s is given by Sohma [123].

In 1940 Kauzmann and Eyring, at the end of their paper on polymer viscosity, suggested that one mechanism of shear thinning was rupture of polymers caused by cleavage of their backbone $\mathrm{C}-\mathrm{C}$ bonds due to mechanical force [14]. They related this to the influence of force on the potential between the bonded C-C atoms as shown in Fig. 12, which illustrates how an applied force linearly reduces the bonding energy

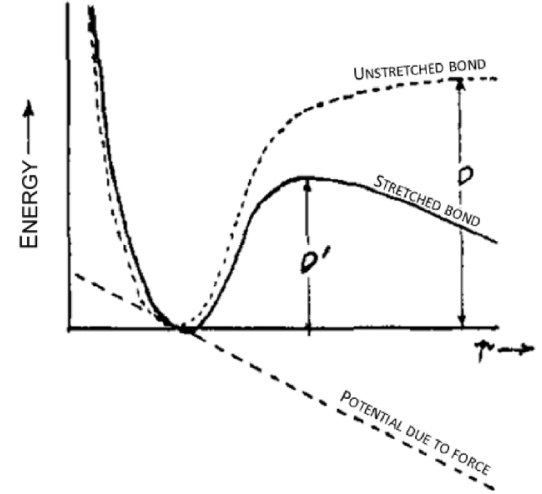

Fig. 12 Influence of applied force on the potential of a C-C polymer bond. Reproduced with permission from [14]. Copyright ACS, 1940.

as the bond is stretched. They then considered how this process might be either reversible, to give temporary shear thinning, or irreversible, to reduce viscosity permanently.

In 1955 Beuche developed a model to describe the rupture of polymer chains under stress in bulk polymers above their glass transition temperature [19]. The rate of segment rupture was given by

$$
\frac{\mathrm{d} n}{\mathrm{~d} t}=\omega v \sum_{i=0}^{N} \mathrm{e}^{-\left(E-V_{i}\right) / k_{\mathrm{B}} T}
$$

where $\omega$ is the segment oscillation frequency, $v$ the number of active polymer chains per unit volume, $N$ the number of segments in a network chain, $E$ the thermal activation energy to break a segment, and $V_{i}$ the elastic energy stored in segment $i$ due to an applied force. This elastic energy was initially defined in terms of the bond stretch, calculated from the length of the bond, the applied tensile stress, and Young's modulus of the polymer, in a way quite similar to Becker's stored elastic energy model of plastic deformation outlined in Section 3.

However, in 1960 Beuche modified his definition of $V_{i}$ to one more similar to Eyring's, with the elastic energy replaced by the product of a force and an activation distance [21]. The probability of chain bond $q$ rupturing was then given by

$$
\frac{\mathrm{d} n}{\mathrm{~d} t} \propto \omega e^{-\left(E-F_{q} \delta\right) / k_{\mathrm{B}} T}
$$

where $\delta$ is the approximately half the distance the bond will stretch before breaking (the activation length) 
and $F_{q}$ is the force on the bond number $q$ from the centre of an active chain segment having total number of links $Z$ given by

$$
F_{q}=F_{\mathrm{o}}\left[1-\left(\frac{4 q^{2}}{Z^{2}}\right)\right]
$$

$F_{\mathrm{o}}$ is thus the maximal force experienced by the central bond and Beuche showed that this was proportional to the product of the shear rate and the bulk viscosity, i.e., the shear stress acting on the whole active chain. A very similar model to Beuche's was proposed by de Gennes in 1974 [124] who also showed that various bond force-distance relationships would all result in an approximately linear decrease of activation energy with applied force.

Perhaps fortunately from the point of view of viscosity modifier polymer stability in lubricants, application of laminar shear subjects a fluid to a combination of rotation and deformation and thus does not impose very large tensile forces. For this reason, fundamental studies of polymer scission in solution are often carried out using elongational flow, where degradation occurs at much lower strain rates. Odell and co-workers studied polymer scission in such flow conditions and showed that the rate of polymer rupture followed a thermally-activated bond scission model very similar to that of Beuche $[125,126]$. They also suggested that most observations of polymer degradation in laminar shear might be due to local turbulence, for example at a capillary inlet. This highlights a key feature that will be discussed later in this paper-that mechanochemistry is dependent on bond stretching and consequent breaking, so that its fundamental study in rubbing contacts requires estimation of tensile forces on molecules from more easily accessible shear forces.

\subsection{Mechanochemistry in Tribology: Recent work}

Polymer degradation as outlined above is a process that takes place in bulk polymers or in solutions under shear. It has also long been recognised that the rubbing of contacting asperities in boundary and mixed lubrication conditions can stimulate chemical reactions at surfaces to form tribofilms. This whole field is generally termed tribochemistry and, as will be discussed in the next section, several different mechanisms have been suggested as drivers of tribochemical reactions. Recognition that stressaugmented thermal activation might cause such reactions at surfaces and the description of this as mechanochemistry, is however quite recent. It appears to have originated to describe the shear-induced removal of atoms, ions, or molecules from surfaces when developing models of wear, as will be outlined in the next section, and only in the last three years has it been formally applied to tribochemical reactions. Since 2015, however, progress has been quite remarkable and stress-augmented thermal activation models are now being used to describe antiwear and extreme pressure (EP) additive reactions as well as polymerisation in rubbing contacts.

In an influential paper in 2015, Gosvami et al. measured the rate of film formation by the antiwear additive zinc dialkyldithiophosphate (ZDDP) on silicon and iron-coated surfaces in a liquid cell AFM at elevated temperature [127]. As shown in Fig. 13, the topography mapping capability of AFM enabled film formation to be monitored as a function of applied load and temperature. An exponential dependence of film growth rate on pressure was observed, and the observed temperature dependence was correlated with a stress-augmented thermal activation model

$$
\Gamma_{\text {growthrate }}=\Gamma_{0} \mathrm{e}^{-\frac{\Delta G_{\text {act }}}{k_{\mathrm{B}} T}}
$$

where $\Gamma$ is the ZDDP film growth rate, $\Gamma_{0}$ a pre-factor and $\Delta G_{\text {act }}$ the free energy activation energy of the rate

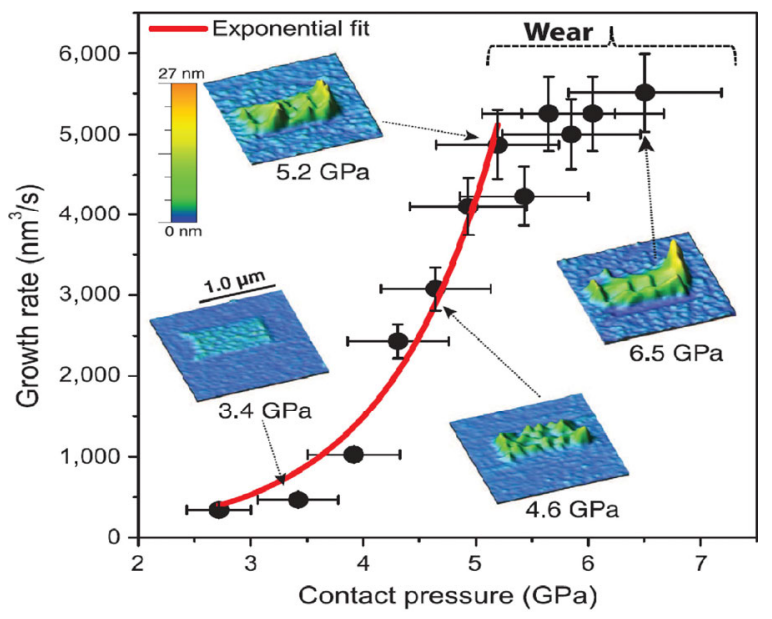

Fig. 13 ZDDP tribofilm growth rate versus contact pressure on a silicon surface. Topography images are after 2,000 cycles. Reproduced with permission from [127]. Copyright Science ASSS, 2015. 
limiting reaction step, given by an expression with a thermal activation and a stress activation term

$$
\Delta G_{\mathrm{act}}=\Delta U_{\mathrm{act}}-\sigma \Delta V_{\mathrm{act}}
$$

Here $\Delta V_{\text {act }}$ is the activation volume and $\sigma$ the driving stress. Gosvami assumed this driving stress to be pressure but, as will be discussed in the next section, it is now believed to be primarily the shear stress (or rather the tensile stress inflicted on the ZDDP molecules via the shear stress). Generally, of course, in boundary lubrication the shear stress will be approximately proportional to the pressure at a given temperature, at least over a limited pressure range.

Tysoe and co-workers studied the tribochemistry of two model sulphur-based extreme pressure (EP) additives, dimethyl and diethyldisulphide, on copper. They investigated each stage of the reaction process: (i) adsorption/reaction of EP molecules on the surface to form alkylthiolate; (ii) the reaction of this alkylthiolate during rubbing to break C-S bonds and release sulphur atoms; (iii) the forced diffusion of these $\mathrm{S}$ atoms into the metal substrate $[128,129]$. By measuring the release of hydrocarbon species using mass spectrometry they were able to monitor the second stage of this process and found that the reaction rates measured during rubbing were consistent with Bell's stress-augmented thermal activation model [129]. Molecular dynamics simulation was also used to examine the likely response of individual methylthiolate $\left(\mathrm{CH}_{3} \mathrm{~S}\right)$ species at the surface during shear. The applied force tilted the molecules and the resulting stretching force promoted breaking of the C-S bond, as shown in Fig. 14 . Comparison of the measured reaction rate and MD predictions indicated that the rate-limiting step for the reaction was methylthiolate decomposing to adsorbed methyl and sulphur species.

Also in 2015, Felts et al. studied the influence of contact stress on the removal of oxygen atoms bonded to graphene by a sliding AFM tip [130]. Since oxygen-free graphene gives much lower friction than graphene coated with bound oxygen, the kinetics of oxygen removal could be monitored by measuring the decrease of lateral force. The results correlated well with a stress-augmented thermal activation model, with an activation volume of $11 \AA^{3}$ and a thermal activation energy of $0.75 \mathrm{eV}$. The authors measured

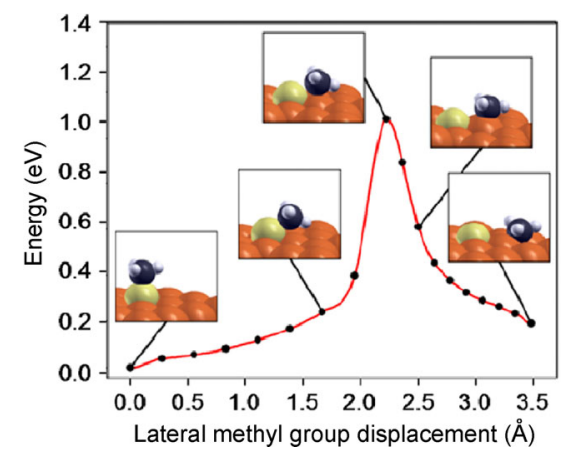

Fig. 14 Profile of calculated energy versus lateral displacement of the methyl group in methyl thiolate on a $\mathrm{Cu}(100)$. Also shown is the evolution in the methyl thiolate to release an adsorbed methyl group. Reproduced with permission from [128]. Copyright ACS, 2015.

tip geometry after sliding and were thus able to obtain good estimates of contact pressure and shear stress. Like Goswami however, they appear to have used normal pressure as the driving stress in their model.

Two recent studies have investigated the effect of contact stress on tribopolymerisation. Yeon et al. investigated the rate of formation of solid reaction product in a sliding silicon oxide on glass contact lubricated by allyl alcohol vapour, using an AFM to monitor the volume of polymerised product [131]. They also carried out parallel MD simulations. Both experiment and model found reaction rate to increase exponentially with pressure and shear stress as shown in Fig. 15.

He and Kim studied the adsorption of, and then rate of formation of polymeric deposit by $\alpha$-pinene, pinane, and n-decane during sliding of stainless steel on stainless steel [132]. They used reflection adsorption IR spectroscopy to measure adsorption and AFM to monitor the volume of solid material produced in and around the contact. Like Yeon et al., they found the rate of polymerisation to depend exponentially on load. The reaction rate for $n$-decane was much slower than for the ring-structured pinene and pinane.

The above experimental work has inspired models that predict tribofilm growth, removal, and consequent friction and wear in rough surface contacts by numerical modeling, with tribofilm formation governed by the local temperature and stress in a time-evolving system [133,134]. The current main limitation of such models is that while film formation rates can be modelled now with reasonable confidence, the processes 

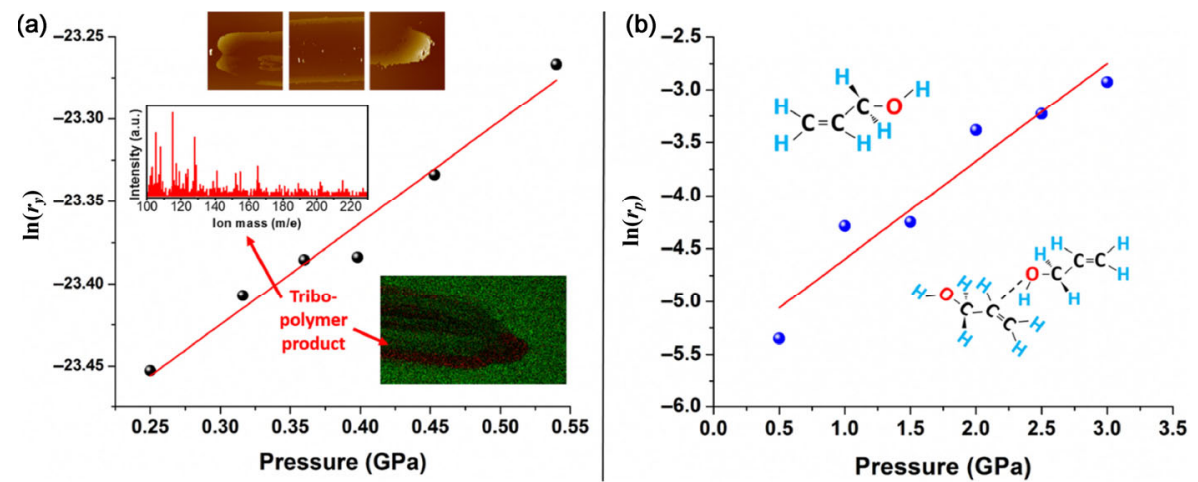

Fig. 15 (a) Rate of measured normalised tribo-polymer yield versus applied contact pressure. Insets are AFM images of a rubbed track, a mass spectrum of tribo-products from ToF-SIMS analysis, and a ToF-SIMS imaging ion map. (b) Rate of tribo-polymer formation predicted from MD simulations. Insets show the structures of an allyl alcohol molecule and an intermediate dimer. Reproduced with permission from [131]. Copyright ACS, 2017.

and equations that describe film removal are not yet at all well understood or reliable.

\subsection{Proof of mechanochemical reaction}

It is clear from the above that the recognition that some tribochemical reactions may be driven by stress according to a stress-augmented thermal activation mechanism is growing very rapidly. A word of caution should be noted however. The principle evidence that a tribochemical reaction is driven by stress, and thus falls into the category of mechanochemistry, is almost always an observed exponential increase in reaction rate with applied pressure or shear stress, in some cases supported by molecular dynamics simulation. This presumes however that other possible drivers are absent and/or such other drivers will not also increase reaction rate exponentially with stress. Historically there are many suggested drivers for tribochemical reactions including, in addition to pressure or shear stress, the following:

- Flash temperature;

- Generation of catalytic surfaces;

- Activation/roughening of surfaces;

- Enhanced mixing due to shear;

- Localised non-Boltzmann energy distributions;

- Triboemission.

The mechanochemical studies described in the previous section [127-132] above all used very low sliding speeds in order to minimise significant flash temperature rises, but it is debatable whether the other possible drivers listed above can be safely discounted. For example, it has already been noted in this review that plastic deformation and fracture of metals and ceramics are exponentially dependent on shear stress, while it has also been found that triboemission stems directly from plastic deformation and/or fracture. Thus the possibility of triboemission may drive the observed reactions that cannot be ignored. Another problem is how to distinguish whether shear stress or pressure is the main driving force since the two are strongly correlated in boundary lubrication.

This problem has recently been addressed by Zhang and Spikes using the additive ZDDP [135]. The underlying problem is that the rubbing contact of asperities is an extremely severe and generally poorly defined process during which it is difficult to know or control the conditions and their effects. The use of an AFM tip alleviates some of these issues but not those relating to nanoscale tip damage processes.

Zhang and Spikes reasoned that if ZDDP reaction is driven by stress then it should occur at very high stress even in the absence of asperity contact: for example, in full film elastohydrodynamic lubrication conditions. They thus monitored ZDDP tribofilm formation in a very high pressure EHD contact where there was no asperity contact. They used a high entrainment speed to generate a thick, separating EHD film, but very low sliding speed to minimise flash temperature. They compared the behaviour of ZDDP in two different solvents, one a polyalphaolefin (PAO) that produced very low EHD friction and thus shear stress and one a traction base fluid with very high EHD friction and shear stress, both giving the same EHD film thickness. As can be seen in Fig. 16, 


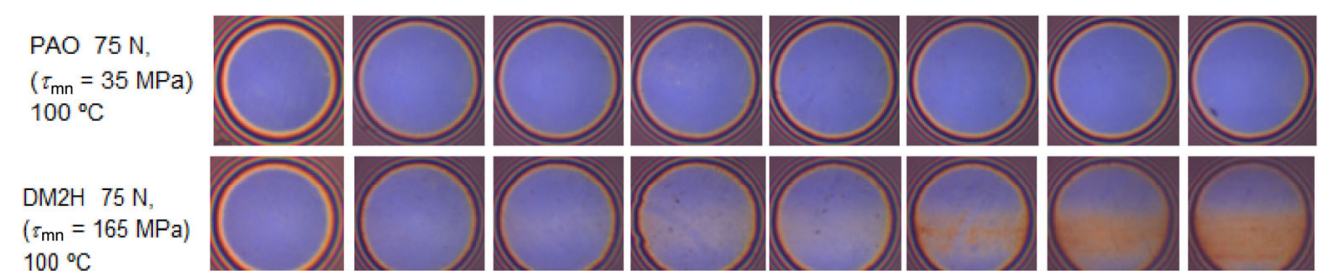

Fig. 16 Comparison of optical interference images for ZDDP solution in PAO and DM2H over a four-hour test at $75 \mathrm{~N}$ and $100{ }^{\circ} \mathrm{C}$ $(U=3 \mathrm{~m} / \mathrm{s}, \mathrm{SRR}=3 \%)[135]$.

they found that ZDDP in the traction base fluid DM2H formed a tribofilm on the rolling-sliding surfaces at high shear stress, but ZDDP at PAO formed no such film at low shear stress even though all other conditions, including pressure, were otherwise identical. From the measured friction it was evident that, depending on the temperature, ZDDP started to form a film when the local shear stress exceeded about $200 \mathrm{MPa}$.

The ZDDP tribofilm increased linearly with time and the dependence of the rate of the film growth on shear stress and temperature agreed well with the stress-augmented thermal activation model.

This study is important because, as well as eliminating all of the alternative possible drivers listed above, leaving only contact stress as the driver, it also demonstrated clearly that the ZDDP reaction was promoted primarily by shear stress and not normal pressure. For the ZDDP in traction base fluid studied, Zhang and Spikes found a thermal activation energy of $53 \mathrm{~kJ} / \mathrm{mol}$ and an activation volume of $180 \AA^{3}$.

\section{Wear}

The final area where stress-augmented thermal activation has been applied in Tribology is in the field of wear. This is closely related to the mechanochemistry outlined above since it is presumed that wear involves the breaking of bonds (ionic, covalent, metallic) and consequent release of one or more particles from a solid sliding surface. In 1997 Dickinson et al. studied the dissolution of calcite into calcium carbonate solution as an AFM tip was rubbed over the calcite surface [136]. He found the dissolution (wear) rate increased exponentially with the applied contact force and fitted the data to a "Zhurkov-Arrhenius" model. Based on the radial tip stress he estimated an activation volume of $44 \AA^{3}$.
In 2000 Kopta and Salmeron investigated the formation of wear scars on mica surfaces rubbed by a $\mathrm{Si}$ tip in an AFM [137]. They found that rubbing caused breakage of $\mathrm{Si}-\mathrm{O}$ bonds in the mica to form defects that developed into $2 \AA$ deep wear scars. They fitted the results to a stress-augmented thermal activation model in which the rate of Si-O bond breaking and thus wear was exponentially dependent on the stress.

Gottsman and Lanz in 2008 studied the wear of cross-linked polyaryletherketone rubbed by a sharp Si tip in an AFM and applied Briscoe and Evans' model of boundary friction to explain the influence of applied load on wear [138]. More recently Jacobs and Carpick rubbed a silicon tip against a diamond flat in vacuum in a transmission electron microscope (TEM) and used the TEM images to measure the change in volume of the tip and thus the wear rate [139]. As shown in Fig. 17, they found this wear rate to vary exponentially with applied mean normal contact stress and attributed this to a stress-augmented thermally activated reaction with thermal activation energy $0.91 \mathrm{eV}$ and an activation volume based on contact pressure of $6.7 \AA^{3}$. This corresponded to an activation volume of $55 \AA^{3}$ if shear stress were considered to be the driver.

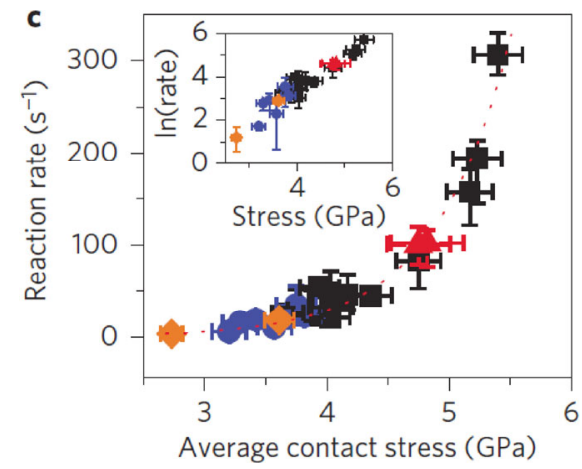

Fig. 17 Influence of contact pressure on the wear rate of pure silicon against diamond in a TEM. Reproduced with permission from [139]. Copyright Nature, 2013. 


\section{General discussion}

\subsection{Connections}

It is striking from all of the research outlined in the previous five sections how the principle of stressaugmented thermal activation links together apparently disparate areas of Tribology. Not only are models of plastic deformation, liquid rheology, friction, tribochemistry, and wear all based on the same underlying concept but also the boundaries between them become blurred. Thus Eyring's model, originally developed to describe liquid viscosity, has been extended and used to describe plasticity, while Prandtl's model spans the whole range of behaviour from crystal plasticity to viscosity as temperature is increased. Kauzmann and Eyring's polymer shear thinning and Shallamach's polymer friction model are based on the formation and breaking of bonds and are thus precursors of mechanochemistry, suggesting that friction and tribochemistry may be interlinked at a fundamental level. Atomic-scale wear and mechanochemistry are also directly related

It is not always clear from the literature how Prandtl's and Eyring's original concepts spread and the extent to which they were independently rediscovered by subsequent researchers. Figure 18 attempts a chart showing the links from researcher to researcher where these are reasonably clear. It is evident that the studies of Eyring and of Zhurkov are the origins of most recent work.

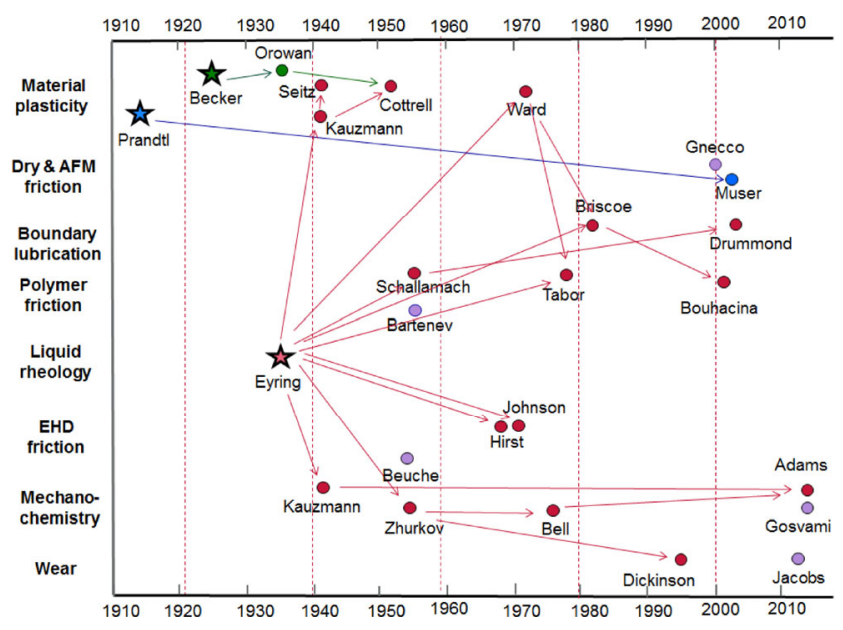

Fig. 18 Timeline showing connections in application of stressaugmented thermal activation.

\subsection{Activation constants}

One point of some confusion in the literature concerns the significance of shear activation volume. Prandtl, Eyring, Schallamach, and Beuche used an activation distance $\Delta x$ in their versions of the rate equation $k=$ $A \mathrm{e}^{-\left(E_{\mathrm{a}}-f \Delta x\right) / k_{\mathrm{B}} T}$, respectively employing the nomenclature, $a, \lambda / 2, \gamma$, and $\delta$ in Eqs. (16), (8), (10), and (11) ( $a$ does not appear explicitly in Prandtl's Eq. (16) but is equal to $A / B)$. These all have unit of length and so, when multiplied by the applied force $f$, provide the work term $f \Delta x$ that promotes the process. In practice, the activation length generally approximates to the distance from the initial valley to the top of the potential energy barrier that must be surmounted for the process to occur. It thus corresponds broadly to half molecular spacing or bond length.

Unfortunately, in experimental work the force on a molecule is not generally known; what is known is the applied shear stress calculated from the friction force divided by the contact area. Thus most experimental researchers have employed rate equations of the form; $k=A \exp \left(-\left(E_{\mathrm{a}}-\tau \Delta v\right) / k_{\mathrm{B}} T\right)$ where $\tau$ is the shear stress and $\Delta v$ is called the shear activation volume. This has units of volume and so when multiplied by stress forms the required work term. It is very simply determined from the gradient of applied stress versus $\log$ (rate), where rate is sliding speed, strain rate, tribofilm formation rate, etc. This gradient is $k_{\mathrm{B}} T / \Delta v$.

It is, however, important to note that $\Delta v$ is not actually a volume but, as should be clear from Eyring's derivation in Section 4.1, the product of the area over which the stress acts multiplied by an activation distance. If the driving stress is a shear stress, this active area corresponds to the cross-sectional area of a molecule or set of molecules in the plane parallel to the direction of stress. In Zhurkov's work, where a tensile stress is applied, the activation area is, of course, orthogonal to the applied stress. This stress may be acting on a single molecule, in which case the active area is the area of one molecule. However, if the interface contains defects, the stress acting on several molecules may focus the resulting force on a single molecule to help drive it over an energy barrier. In this case the active area will be larger, and possibly much larger, than a single molecule, as is the case in the deformation of metals. 
It is of interest to compare the various values of shear activation volume determined by researchers. Eyring stresses are typically in the range 5 to $50 \mathrm{MPa}$. From Eyring's model, (Eqs. (22) and (23)), Eyring stress is equivalent to $2 k_{\mathrm{B}} T / \lambda_{2} \lambda_{3} \lambda$ where $\lambda_{2} \lambda_{3}$ is the area of a molecule over which the stress acts and $\lambda$ is the distance a molecule or molecular segment moves in a jump (twice the activation length). $\lambda_{2} \lambda_{3} \lambda / 2$ is thus the activation volume and, over the Eyring stress range above, it spans 100 to $1,000 \AA^{3}$. For approximately spherical molecules, if the shear activation originates from the force on individual molecules, the shear activation volume will be approximately half that of the cube containing a molecule, so this can be compared to the molecular volume. A hydrocarbon lubricant of average MW 400 will have a molar volume of $c a$ $350 \mathrm{~cm}^{3}$ and so a mean molecular volume of $\mathrm{ca} 580 \AA^{3}$. Of course because of free volume, the actual occupied molecular volume will be somewhat smaller than this. For non-spherical molecules, if we arbitrarily assume a thickness perpendicular to the shear plane (Eyring's $\lambda_{1}$ ) of $10 \AA$, we obtain a molecular area on which the shear stress acts of 20 to $200 \AA^{2}$. As noted by Hirst and Moore [54] these are not unreasonable values, in that for polymeric molecules the flow unit is likely to be a polymer segment, while for molecules having short side chains some interlocking is likely so the flow unit may well be more than one molecule.

In studies on boundary friction, Briscoe and Evans [84] and Chugg and Chaudri [85] estimated shear activation volumes of the fatty acids studied of approximately 1,500 to $6,000 \AA^{3}$. They suggested that this was equivalent to about 2-6 molecular volumes and that a small number of molecules were therefore combined as flow units. However, the authors compared molecular volume with activation volume and this is probably not appropriate for vertically-oriented molecules. As discussed above, the activation volume is generally considered to be the area of the molecule times its activation length. The area of a fatty acid head group is around $20 \AA^{2}$ [140] from which its activation length will be about $2.5 \AA$ in a close packed monolayer. This suggest an activation volume of $c a 50 \AA^{3}$, implying that 30 to 120 molecules may be involved in experiencing the driving stress. This raises the possibility of slip resulting from localised dislocation motion, as deduced for plastic flow of metals.
In mechanochemistry, Gosvami et al. [127] calculated an activation volume for ZDDP tribofilm formation of $3.8 \AA^{3}$ but this value presumed the driving force was pressure. If it were shear stress then, assuming a friction coefficient of 0.1 , the shear activation volume would be $38 \AA^{3}$. Zhang and Spikes [135] determined an activation volume value for ZDDP of $180 \AA^{3}$. It is, of course, extremely unlikely that the two ZDDPs studied by Gosvami and Zhang were the same. For breaking of the Si-O bond Felts [130] found an activation volume of $11 \AA^{3}$ but it is not clear whether this was calculated on the basis of pressure or shear stress as the driver. Yeon et al. [131] calculated an activation volume of $7.8 \AA^{3}$ for tribopolymerisation of allyl alcohol. If we assume an activation distance of about 1 to $2 \AA$, corresponding to a covalent bond length, these values yield activation areas that are reasonably representative of the areas of single molecules.

As already mentioned, the values of activation volume estimated for plastic deformation of metals are much larger than for most other applications of stress-augmented thermal activation. This is because plastic deformation involves the motion under stress of 2D structural entities consisting of many atoms, so the activation dimensions are considerably larger. They can thus be regarded as meso-scale stress-augmented thermal activation models. This is especially evident in Becker's [6] model which involves a bulk property of the material, its elastic modulus, but is also the case for Kauzmann's model [13].

\subsection{Forms of stress}

While it may be reasonable to use shear stress in stress-activated models of friction, rheology, and plastic deformation, it is probably less appropriate in the context of mechanochemistry. Here it is generally believed that tensile forces act on atom-atom bonds to promote bond breaking, so rate equations should really be based on tensile stress or force. Unfortunately, we currently do not have reliable ways to measure the tensile forces imparted to lubricant molecules from the shear stresses present in asperity-asperity or EHD contacts. The rate equations employed in Refs. [127-132] all implicitly assume proportionality between shear force and tensile force. The issue is complicated by the fact that we need to consider the tensile forces 
experienced by molecular bonds at surfaces, which may well be different from those in the bulk. Thus, based on their modelling work, Yeon et al. noted [131]:

"It appears that anchoring one molecule to the surface helps transfer the mechanical force or action from the solid surface to the other molecule being reacted."

Another stress that should be considered is normal pressure. As outlined in Section 5, some researchers include a pressure term in the stress-activated rate equation, as shown in Ward's Eq. (18) and Briscoe and Evans' Eq. (31). Here the applied hydrostatic pressure is assumed to influence the rate of the process via a pressure activation volume. For friction this term is generally considered to be negative since slip is assumed to require a local, albeit very small, motion away from the counter-surface against the applied pressure. Hirst and Moore also included a negative pressure activation term in addition to a positive shear activation one in their EHD friction analysis [54]. Nowadays in EHD this tends to be subsumed in the highly pressure-sensitive Newtonian viscosity term as in Eqs. (24) and (27) while the Eyring stress is generally also found to increase linearly with pressure.

In mechanochemistry, the sign of a pressure term should depend on the volume change associated with bond breaking and Gosvami et al. implicitly assume there to be a reduction in volume for ZDDP film formation by their use of pressure as the reaction driver [127]. It should also be noted that although shear stress has been subsequently been shown to drive ZDDP tribofilm formation [135] this does not, of course, mean there might not also be a pressure activation term, and further work needs to be done to establish this. This would be very difficult to do in boundary lubrication conditions where normal pressure and shear stress are coupled, but should be feasible using Zhang and Spikes EHD approach [135], where they can be varied independently by changing the solvent friction.

\subsection{Forward and reverse reactions}

It should be clear from the above that although both the Prandtl and Eyring models of stress activation include both forward and reverse terms, to give, in Eyring's case the dependence of rate on $\sinh$ (stress), in practice in most experimental studies only the forward reaction appears relevant in that the rate is found to depend exponentially on stress. There are a few exceptions, notable in EHD rheology [54, 59], very high temperature plastic deformation [32, 141], and very low speed friction modelling [101], but generally the reverse term is negligible. This can be understood by considering Eq. (7) based on a stress activation volume

$$
k_{\text {net }} \propto \sin h\left(\tau \Delta v / k_{\mathrm{B}} T\right)
$$

If $\tau \Delta v / k_{\mathrm{B}} T$ is greater than about 1.5 then the $\sin h()$ term becomes practically equal to $0.5 \exp \left(\tau \Delta v / k_{\mathrm{B}} T\right)$, so that rate increases exponentially with stress. The $\sinh ($ ) dependence will thus only become evident if $\tau \Delta v / k_{\mathrm{B}} T$ is below this level. At a representative temperature of $60{ }^{\circ} \mathrm{C}$ this would imply that $\tau \Delta v$ should be below about $5 \times 10^{-21} \mathrm{~m}$. In both EHD and boundary friction, activation volumes are typically 100 to $6,000 \AA^{3}$, suggesting that then $\sin h()$ dependence of rate on stress would be evident at shear stresses below somewhere between 1 and $50 \mathrm{MPa}$. These shear stresses are commonly present in relatively low load EHD contacts, so a $\sin h$ dependence may be seen, but are not usually found in boundary friction, where shear stresses at asperity contacts are generally much higher.

In mechanochemistry of course, another factor militating against a significant reverse reaction rate is that the thermal activation energy barrier is generally lower in the forward than the reverse direction.

\subsection{Force-displacement relationships}

So far, almost all of this review has assumed the simplest form of the stress-augmented thermal activation equation, i.e., the applied force reduces the stress activation energy linearly with displacement, $x$, so $k_{\mathrm{f}}=A \mathrm{e}^{-\left(E_{\mathrm{a}}-f \Delta x\right) / k_{\mathrm{B}} T}$. This is, however, only an approximation and two subtly different refinements have been applied: one by adopting more accurate solutions of the balance between the applied force and the potential field with which it interacts, the second allowing the position of the maximum and minimum of the potential field to be shifted by the applied force.

Prandtl noted that a linear dependence of activation energy on displacement, $U_{1}=A-B x$, was only an approximation in his model and that a more accurate 
expression would allow it to vary parabolically so that $U_{1}=$ const. $(a-x)^{3 / 2}$ [2]. He showed how an approximate solution could be derived for the jump probability and thus the dependence of total force on sliding velocity and temperature using this relationship, but analysis was complicated and limited [2].

Gnecco et al. [142] applied both linear and parabolic expressions to sliding AFM models and found that the linear form of the stress activation energy (Eq. (36)) was satisfactory for low values of force but that a parabolic form as in Eq. (44) should be used when the force was close to its critical jump value, $F^{*}$

$$
\Delta E^{+}(F)=\mu\left(F^{*}-F\right)^{3 / 2}
$$

As well refinements in the potential versus displacement solution, the effect of applied force on the overall shape of the potential field has been considered, in particular allowing for locations of the minimum and maximum of the potential curve and thus the stress activation distance to vary with applied force. This was considered in the context of plasticity in 1975 when Kocks et al. used a Taylor expansion to examine the impact of allowing the total activation energy to vary non-linearly with both stress, $\sigma$, and apparent activation area [38]. They concluded that a linear dependence of total activation energy on both stress and activation area was valid so long as the stress did not approach the maximum plane glide resistance.

The influence of applied force on the activation distance has also been considered in the context of mechanochemistry in what is generally known as the extended Bell model [143]. This takes account of the applied force shifting both the potential minimum and maximum as shown schematically in the potential diagrams in Fig. 19, where $\xi$ is the reaction path coordinate, the solid line is the potential when no force is present and the dashed line shows the effect of applied force. According to extended Bell theory, the total activation energy $V(F)$ at applied force $F$ is given by

$$
V(F)=V(0)-F \Delta x-\frac{F^{2}}{2}\left(\chi_{\mathrm{T}}-\chi_{\mathrm{I}}\right)
$$

where $V(0)$ is the thermal activation energy, and $\chi_{\mathrm{I}}$ and $\chi_{\mathrm{T}}$ are the compliances of the molecule in its

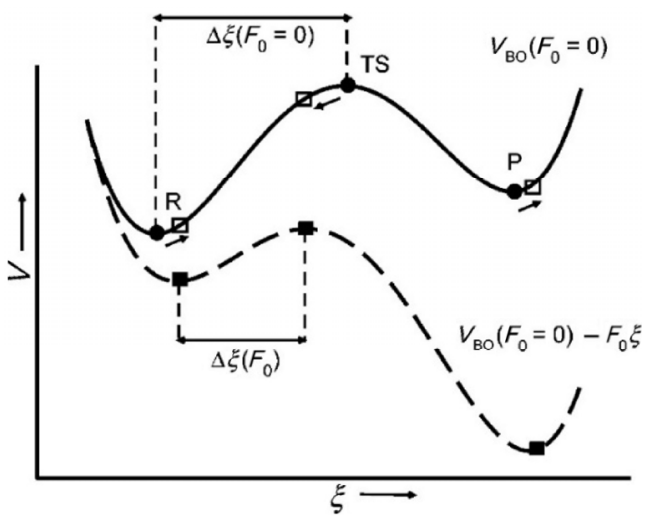

Fig. 19 Effect of a constant stretching force on a given reaction profile. Reproduced with permission from [109]. Copyright ACS, 2012.

initial and transitions state (i.e., at the minimum and maximum positions of the potential barrier). The product $F \chi$ is the distance these positions move due to the applied force.

Furlong et al. have explored the impact of the shape of the potential field on sliding friction behaviour [144], while Tysoe has recently provided a detailed discussion of the influence of applied stress on activation barriers and interpreted experimental mechanochemical results using advanced expressions for the activation energy [145].

\subsection{Prandtl and Eyring}

It is of interest to compare Prandtl's and Eyring's models. This was done in some detail in Ref. [16] and so is only briefly summarised here. Although both are based on stress-augmented thermal activation they are fundamentally different in two aspects. Firstly, Prandtl considers the transition across a potential barrier of a finite number of particles. This means that his rate of transition varies as the population of particles on either side of the barrier changes. This gives the $\mu$ and $(1-\mu)$ pre-factors in Prandtl's Eqs. (14) and (15) and it is these that make solution of these equations difficult. By contrast, Eyring essentially assumes a very large ensemble of particles, so large that the number of particles available to transit is invariant and effectively the same in both directions. This results in Eq. (7) where the forward and reverse exponents appear without pre-factors, making the equation much more tractable. It is perhaps a pity 
that Prandtl did not realise this simplification but his use of varying population levels either side of the transition did enable him to apply his model to stress relaxation as well as steady state plastic flow.

Models of AFM friction illustrate the extreme of Prandtl's approach in that only one particle (the AFM tip) traverses an energy barrier [91]. Schallamach's revised model which involves the formation and breaking of junctions also has similarities with Prandtl's approach since it takes account of the varying fraction of surviving junctions during sliding [76].

The second main difference is that Prandtl assumes that his sliding particles are elastically constrained so that their displacement is proportional to the applied force. Eyring however implicitly assumes that the constraining force on his particles is independent of their displacement-in effect being constrained by a constant force spring. Eyring's assumption has been embedded in most subsequent work, but Becker's, Schallamach's, and AFM friction models involve elastic constraint.

\subsection{A caveat}

This review has concerned the application of the concept of stress-augmented thermal activation to develop atomic-scale models of plasticity, friction, tribochemistry, and wear. Before concluding, it is important to note that, while evidently operative in many cases, this should not be regarded as the only mechanism underlying these phenomena. Thus while many processes exhibit the relationship stress $\propto \log$ (rate), others do not. For example in EHD at high strain rates most simple liquids show friction that increases with $\log$ (strain rate) in isothermal conditions, but traction fluids reach a constant value [146]. In boundary friction Briscoe and Evans found that fatty acid films showed friction proportional to $\log$ (sliding speed) but this was not the case for fatty acid soaps [84]. Campen et al. showed that the friction-speed response depended on whether the adsorbing surfactants could form close-packed monolayers [87]. Drummond et al. showed that friction as only proportional to log(sliding speed) up to a critical speed [88]. These suggest that the applicability of stress-augmented thermal activation has a finite scope. Of particular interest is to understand when and why it ceases to be applicable rather than only studying conditions under which it seems to be prevalent.

It is also possible that other mechanisms that control rheology and friction may give observed stress $\propto \log$ (rate) behaviour. It has already been noted that network-based rheology models might do this in some cases in the context of EHD friction. The problem then becomes to develop experimental ways of distinguishing between mechanisms. This was done in the field of tribochemistry where a designed experimental approach showed that shear stress (and not many alternative drivers) almost certainly controls ZDDP reaction rate [135]. But this does not mean that all tribochemical reactions are driven by stress. Indeed, almost all the factors listed in Section 6.4 are likely to, and in most cases have been shown to, accelerate tribofilm formation under some conditions, the only exception to date being non-Boltzmann energy distributions.

In summary, stress-augmented thermal activation almost certainly plays a key role in controlling some, and probably many, friction, tribochemical, and wear processes but the range of conditions over which it is important is finite and other processes may compete with or complement it and cannot safely be ignored.

\subsection{Future directions}

How is the concept of stress-augmented thermal activation likely to develop within Tribology in the near future? Clearly, from the scientific viewpoint, the most important issue is to fully test that the underlying principle of atomic and molecular response due to a combination of temperature and stress is valid. Experiments at the individual atomic and molecular scale are difficult, so the most promising approach is to use molecular dynamics simulation to test whether the patterns of behaviour assumed in stress-augmented thermal activation models do actually occur, i.e., when shear and sliding boundary conditions are imposed, are atoms or molecules with thermal energy induced by shear forces to translate past their neighbours to an extent that matches Prandtl's or Eyring's model? And when a very thin molecular layer is sheared, do the forces transmit to molecules at surfaces stretch and break their covalent bonds in the fashion by mechanochemistry? Crucially molecular dynamics is 
able to determine the actual shear and tensile forces experienced by molecules in sheared systems and relate these to the overall ensemble shear stress. A few such simulations of this types have already been made and many more are likely in the near future.

At the experimental level things are more difficult. In the area of EHD friction, one problem has always been that, while we know the shear forces on the two bounding solid surfaces and also the velocities of these surfaces, this does not tell us how the fluid film shears between them. Does the velocity within the fluid vary linearly across the film (Couette flow) as is commonly assumed when estimating the shear rate, or during shear thinning is there shear localisation such as plug flow or a slip band? Without such information it is not possible to test Eyring's shear thinning model fully, or indeed other rheological models. This issue may be addressed via molecular simulation [69] but a recent, complementary experimental approach is phosphorescence imaging, where the velocity of the fluid across a thin EHD film can be profiled from the convection of a small, laser-excited column of fluid within the film [147].

In terms of tribofilm formation, it is very likely that studies will be made in the next few years on many more additive systems to test whether film formation rate depends on stress and temperature as predicted by stress-augmented thermal activation. Unfortunately, such measurements do not resolve the problem of distinguishing the impact of shear stress and normal pressure since, as discussed in Section 8.3, these are always strongly coupled. They can, however, be decoupled using the EHD film approach outlined in Section 6.4 and this approach should provide useful information about mechanochemistry in general, and the separate influences of shear stress and pressure in particular, over the next few years. Another possibility is to measure covalent bond stretch in high shear stress contacts using a spectroscopic method. This has already been done in some solid-state systems, e.g., Ref. [148], but is technically difficult for very thin films because of the limited number of active centres, and thus the very weak signal. Another possible avenue would be the application of nano-manipulation methods, already developed for chemical studies [109], to investigate the properties of single lubricant additive molecules on surfaces, such as the strength of metal-ligand bonds.

In the context of friction, single contact studies would appear to be a useful way forward, but ideally using stiffer supports than those usually employed in an AFM. Such studies, in parallel with very welldefined surfaces such as Langmuir Blodgett films or single crystals, should help determine the boundaries of stress-induced thermal activation.

\section{Conclusions}

This paper has described the origins of stressaugmented thermal activation and has outlined its application to Tribology. This concept has provided the basis of a series of closely-related, atomic-, and molecular-based models to explain and predict plastic flow, EHD friction, solid-solid friction, tribochemistry, and atomic-scale wear. The key prediction of these models is that the rate of the process involved (flow, sliding, covalent bond breaking, or atom/ion removal from a surface) increases exponentially with shear stress, so that the stress increases with the logarithm of the rate. For example, it is very often found that friction rises logarithmically with sliding speed in EHD, polymer, and boundary friction while tribofilm formation and polymer scission rates increase exponentially with shear stress.

One area of application that is growing very rapidly is tribochemistry, where several recent studies have suggested that the formation of several types of reaction films on rubbed surfaces is driven by stress-augmented thermal activation. This type of tribochemistry is now often termed mechanochemistry. In principle, both normal stress (pressure) and shear stress might drive mechanochemical reactions and one problem in such studies is to distinguish between the effects of these on reaction rate, since pressure and shear stress are general coupled in dry or boundary lubricated sliding conditions. A recent study has shown how these can be decoupled using a thick film rolling-sliding EHD contact with solvents having very different friction coefficients and this has indicated that shear stress is the key driver in ZDDP tribofilm formation.

The fundamental importance of the stress-augmented thermal activation concept is that it provides a link between the nano-scale behaviour of individual atoms 
and molecules and macro-scale responses such as friction and wear. Some future possibilities in research on stress-augmented thermal activation have been outlined. Modern non-equilibrium molecular dynamics simulation methods offer the possibility of validating and exploring further the implications of this mechanism and, indeed, converting such simulations into useful, generalised tools for predicting how molecular structure and surface properties influence macroscale performance. In future, such tools hold the promise of enabling the design of molecules and surfaces for optimised friction and wear performance much more efficiently and rapidly than is currently possible by trial and error.

Finally, it is concluded that stress-augmented thermal activation offers a guiding and unifying principle for explaining many of the processes that take place in sliding tribological contacts.

Open Access: The articles published in this journal are distributed under the terms of the Creative Commons Attribution 4.0 International License (http:// creativecommons.org/licenses/by/4.0/), which permits unrestricted use, distribution, and reproduction in any medium, provided you give appropriate credit to the original author(s) and the source, provide a link to the Creative Commons license, and indicate if changes were made.

\section{References}

[1] Karman T V. Physikalische Grundlagen der Festigkeitslehre, Section 24 767-770, Sept. 1914. Art 31, of Mechanics of deformable bodies in Enzyklopädie der mathematischen Wissenschaften mit Einschluss ihrer Anwendungen, Vol. IV, Part 4, C, eds. Felix Klein and Conrad Muller, Leipzig, 1907-1914.

[2] Prandtl L. Ein Gedankenmodell zur kinetischen Theorie der festen Körper. Z Angew Math Mech 8(2): 85-106 (1928)

[3] Eyring H. Viscosity, plasticity, and diffusion as examples of absolute reaction rates. J Chem Phys 4(4): 283-291 (1936)

[4] Moore W J. Physical Chemistry. 4th ed. London (UK): Longman Green \& Co., 1963.

[5] Ewell R H. The reaction rate theory of viscosity and some of its applications. J Appl Phys 9(4): 252-269 (1938)

[6] Becker R. Über die plastizität amorpher und kristalliner fester körper. Phys Z 26: 919-925 (1925)
[7] Kincaid J F, Eyring H, Stearn A E. The theory of absolute reaction rates and its application to viscosity and diffusion in the liquid state. Chem Rev 28(2): 301-365 (1941)

[8] Glasstone S, Laidler K J, Eyring H. Theory of Rate Processes. New York (USA): McGraw-Hill Inc., 1941.

[9] Ewell R H, Eyring H. Theory of the viscosity of liquids as a function of temperature and pressure. J Chem Phys 5(9): 726-736 (1937)

[10] Powell R E, Eyring H. Mechanisms for the relaxation theory of viscosity. Nature 154(3909): 427-428 (1944)

[11] Eyring H, Ree T, Hirai N. The viscosity of high polymersThe random walk of a group of connected segments. Proc Nat Acad Sci USA 44(12): 1213-1217 (1958)

[12] Moore Jr W J, Eyring H. Theory of the viscosity of unimolecular films. J Chem Phys 6(7): 391-394 (1938)

[13] Kauzmann W. Flow of solid metals from the standpoint of the chemical-rate theory. Trans Am Inst Min Metall Eng 143: 57-83 (1941)

[14] Kauzmann W, Eyring H. The viscous flow of large molecules. $J$ Amer Chem Soc 62(11): 3113-3125 (1940)

[15] Tobolsky A, Eyring H. Mechanical properties of polymeric materials. J Chem Phys 11(3): 125-134 (1943)

[16] Spikes H A, Tysoe W. On the commonality between theoretical models for fluid and solid friction, wear and tribochemistry. Tribol Lett 59(1): 21 (2015)

[17] Schallamach A. The velocity and temperature dependence of rubber friction. Proc Phys Soc B 66(5): 386-392 (1953)

[18] Schallamach A. Friction and abrasion of rubber. Wear 1(5): 384-417 (1958)

[19] Bueche F. Tensile strength of plastics above the glass temperature. J Appl Phys 26(9): 1133-1140 (1955)

[20] Bueche F. Tensile strength of plastics below the glass temperature. J Appl Phys 28(7): 784-787 (1957)

[21] Bueche F. Mechanical degradation of high polymers. J Appl Polym Sci 4(10): 101-106 (1960)

[22] Zhurkov S N, Narzullaev B N. Time dependence of the strength of solids. Zh Tekh Fiz 23(10): 1677-1689 (1953)

[23] Zhurkov S N, Tomashevsky É E. Investigation of the strength of solids. Zh Tekh Fiz 25(1): 66-73 (1955)

[24] Zhurkov S N. Kinetic concept of the strength of solids. Int J Fract Mech 1(4): 311-322 (1965)

[25] Zhurkov S N, Korsukov V E. Atomic mechanism of fracture of solid polymers. J Polym Sci Part B Polym Phys 12(2): 385-398 (1974)

[26] Andrade E N D C, Roscoe R. Glide in metal single crystals. Proc Phys Soc 49(2): 152-177 (1937)

[27] Orowan E. Zur TemperaturabhÄngigkeit der Kristallplastizität. Zeitschr Phys 102(1-2): 112-118 (1936)

[28] Orowan E. Problems of plastic gliding. Proc Phys Soc 52(1): 8-22 (1940) 
[29] De Bruyne N A. Note on viscous and plastic flow. Proc Phys Soc 53(3): 251-257 (1941)

[30] Seitz F, Read T A. Theory of the plastic properties of solids. III. J Appl Phys 12(6): 470-486 (1941)

[31] Gemant A. Frictional phenomena. XI: C-solids. J Appl Phys 13(11): 688-696 (1942)

[32] Feltham P. Influence of structure on the plastic flow of steel above the A3-point. Nature 169(4310): 976(1952)

[33] Mott N F. Dislocations, work-hardening and creep. Nature 175(4452): 365-367 (1955)

[34] Seeger A. CXXXII. The generation of lattice defects by moving dislocations, and its application to the temperature dependence of the flow-stress of F.C.C. crystals. London Edinburgh Dublin Philos Mag J Sci 46(382): 1194-1217 (1955)

[35] Schoeck G. The activation energy of dislocation movement. Phys Status Solidi (b) 8(2): 499-507 (1965)

[36] Gibbs G B. The thermodynamics of thermally-activated dislocation glide. Phys Status Solidi (b) 10(2): 507-512 (1965)

[37] Cottrell A H. The time laws of creep. J Mech Phys Solids 1(1): 53-63 (1952)

[38] Kocks U F, Argon A S, Ashby M F. Thermodynamics and Kinetics of Slip. Oxford, New York (USA): Pergamon Press, 1975.

[39] Antolovich S D, Armstrong R W. Plastic strain localization in metals: origins and consequences. Prog Mater Sci 59: 1-160 (2014)

[40] Pollet J C, Burns S J. Thermally activated crack propagation-Theory. Int J Fract 13(5): 667-679 (1977)

[41] Zerilli F J, Armstrong R W. The effect of dislocation drag on the stress-strain behavior of F.C.C. metals. Acta Metall Mater 40(8): 1803-1808 (1992)

[42] Haward R N, Thackray G. The use of a mathematical model to describe isothermal stress-strain curves in glassy thermoplastics. Proc Roy Soc A Math Phys Eng Sci 302(1471): 453-472 (1968)

[43] Ward I M. Review: The yield behaviour of polymers. J Mater Sci 6(11): 1397-1417 (1971)

[44] Frisch D, Eyring H, Kincaid J F. Pressure and temperature effects on the viscosity of liquids. J Appl Phys 11(1): 75-80 (1940)

[45] Spikes H A, Zhang J. History, origins and prediction of elastohydrodynamic friction. Tribol Lett 56(1): 1-25 (2014)

[46] Ree T, Eyring H. Theory of non-Newtonian flow. I. Solid plastic system. J Appl Phys 26(7): 793-800 (1955)

[47] Ree F, Ree T, Eyring H. Relaxation theory of transport problems in condensed systems. Ind Eng Chem 50(7): 1036-1040 (1958)
[48] Maron S H, Pierce P E. Application of Ree-Eyring generalized flow theory to suspensions of spherical particles. J Colloid Sci 11(1): 80-95 (1956)

[49] Kumar P, Khonsari M M, Bair S. Full EHL simulations using the actual Ree-Eyring model for shear-thinning lubricants. J Tribol 131(1): 011802 (2009)

[50] Smith F W. The effect of temperature in concentrated contact lubrication. ASLE Trans 5(1): 142-148 (1962)

[51] Plint M A. Traction in elastohydrodynamic contacts. Proc Inst Mech Eng 182(1): 300-306 (1967)

[52] Hirst W, Moore A J. Non-Newtonian behaviour in elastohydrodynamic lubrication. Proc Roy Soc A Math Phys Eng Sci 337(1608): 101-121 (1974)

[53] Hirst W, Moore A J. Elastohydrodynamic lubrication at high pressures. Proc Roy Soc A Math Phys Eng Sci 360(1702): 403-425 (1978)

[54] Hirst W, Moore A J. Elastohydrodynamic lubrication at high pressures II. Non-Newtonian behaviour. Proc Roy Soc A Math Phys Eng Sci 365(1723): 537-565 (1979)

[55] Hirst W, Moore A J. The effect of temperature on traction in elastohydrodynamic lubrication. Philos Trans Roy Soc A Math Phys Eng Sci 298(1438): 183-208 (1980)

[56] Tevaarwerk J, Johnson K L. A simple non-linear constitutive equation for elastohydrodynamic oil films. Wear 35(2): 345-356 (1975)

[57] Johnson K L, Tevaarwerk J L. Shear behaviour of elastohydrodynamic oil films. Proc Roy A Math Phys Eng Sci 356(1685): 215-236 (1977)

[58] Hirst W, Richmond J W. Traction in elastohydrodynamic contacts. Proc Inst Mech Eng Part C J Mech Eng Sci 202(2): 129-144 (1988)

[59] Evans C R, Johnson K L. The rheological properties of elastohydrodynamic lubricants. Proc Inst Mech Eng Part C J Mech Eng Sci 200(2): 303-312 (1986)

[60] Conry T F. Thermal effects on traction in EHD lubrication. J Lubr Technol 103(4): 533-538 (1981)

[61] Muraki M. Molecular structure of synthetic hydrocarbon oils and their rheological properties governing traction characteristics. Tribol Int 20(6): 347-354 (1987)

[62] Sui P C, Sadeghi F. Non-Newtonian thermal elastohydrodynamic lubrication. J Tribol 113(2): 390-396 (1991)

[63] Muraki M, Dong D. Derivation of basic rheological parameters from experimental elastohydrodynamic lubrication traction curves of low-viscosity lubricants. Proc Inst Mech Eng Part J J Eng Sci 213(1): 53-61 (1999)

[64] Sharif K J, Morris S J, Evans H P, Snidle R W. Comparison of non-Newtonian EHL models in high sliding applications. Tribol Ser 39: 787-796 (2001) 
[65] Sharif K J, Evans H P, Snidle R W, Newall J P. Modeling of film thickness and traction in a variable ratio traction drive rig. Trans J Tribol 126(1): 92-104 (2004)

[66] Bou-Chakra E, Cayer-Barrioz J, Mazuyer D, Jarnias F, Bouffet A. A non-Newtonian model based on Ree-Eyring theory and surface effect to predict friction in elastohydrodynamic lubrication. Tribol Int 43(9): 1674-1682 (2010)

[67] Gutzow I, Dobreva A, Schmelzer J. Rheology of nonNewtonian glass-forming melts: Part I Flow-stress relations. J Mater Sci 28(4): 890-900 (1993)

[68] Spikes H A. Comment on: Rheology of an ionic liquid with variable carreau exponent: A full picture by molecular simulation with experimental contribution, by Nicolas Voeltzel, Philippe Vergne, Nicolas Fillot, Nathalie Bouscharain, Laurent Joly, Tribology Letters (2016) 64: 25. Tribol Lett 65(2): 72 (2017)

[69] Ewen J P, Gattinoni G, Zhang J, Heyes D M, Spikes H A, Dini D. On the effect of confined fluid molecular structure on nonequilibrium phase behaviour and friction. Phys Chem Chem Phys 19(27): 17883-17894 (2017)

[70] Jadhao V, Robbins M O. Probing large viscosities in glassformers with nonequilibrium simulations. Proc Natl Acad Sci USA 114(30): 7952-7957 (2017)

[71] Voeltzel N, Vergne P, Fillot N, Bouscharain N, Joly L. Rheology of an ionic liquid with variable Carreau exponent: A full picture by molecular simulation with experimental contribution. Tribol Lett 64(2): 25 (2016)

[72] Voeltzel N, Vergne P, Fillot N, Bouscharain N, Joly L. Reply to the Comment on 'Rheology of an ionic liquid with variable Carreau exponent: A full picture by molecular simulation with experimental contribution'. Tribol Lett 65(2): 73 (2017)

[73] Miller R, Wüstneck R, Krägel J, Kretzschmar G. Dilational and shear rheology of adsorption layers at liquid interfaces. Colloid Surf A 111(1-2): 75-118 (1996)

[74] Bartenev G M. On the theory of dry friction of rubber. Dokl Akad Nauk SSSR 96(6): 1161-1164 (1954)

[75] Bartenev G M, El'kin A I. Friction properties of high elastic materials. Wear 8(1): 8-21 (1965)

[76] Schallamach A. A theory of dynamic rubber friction. Wear 6(5): 375-382 (1963)

[77] Bartenev G M, Lavrentjev V V, Konstantinova N A. The actual contact area and friction properties of elastomers under frictional contact with solid surfaces. Wear 18(6): 439-448 (1971)

[78] Schallamach A. How does rubber slide? Wear 17(4): 301312 (1971)

[79] Steijn R P. Sliding experiments with polytetrafluoroethylene. ASLE Trans 11(3): 235-247 (1968)
[80] Chernyak Y B, Leonov A I. On the theory of the adhesive friction of elastomers. Wear 108(2): 105-138 (1986)

[81] Amuzu J K A, Briscoe B J, Tabor D. The shear properties of poly (N-alkyl methacrylates) in concentrated contacts. ASLE Trans 20(2): 152-160 (1977)

[82] Briscoe B J, Tabor D. Shear properties of thin polymeric films. J Adhes 9(2): 145-155 (1978)

[83] Bouhacina T, Aimé J, Gauthier S, Michel D, Heroguez V. Tribological behavior of a polymer grafted on silanized silica probed with a nanotip. Phys Rev B 56(12): 7694-7703 (1997)

[84] Briscoe B J, Evans D C B. The shear properties of Langmuir-Blodgett layers. Proc Roy Soc A Math Phys Eng Sci 380(1779): 389-407 (1982)

[85] Chugg K J, Chaudhri M M. Boundary lubrication and shear properties of thin solid films of dioctadecyl dimethyl ammonium chloride (TA 100). J Phys D Appl Phys 26(11): 1993-2000 (1993)

[86] Ingram M, Noles J, Watts R, Harris S, Spikes H A. Frictional properties of automatic transmission fluids: Part I-measurement of friction-sliding speed behaviour. Tribol Trans 54(1): 145-153 (2011)

[87] Campen S, Green J H, Lamb G D, Atkinson D, Spikes H. On the increase in boundary friction with sliding speed. Tribol Lett 48(2): 237-248 (2012)

[88] Drummond C, Israelachvili J, Richetti P. Friction between two weakly adhering boundary lubricated surfaces in water. Phys Rev E 67(6): 066110 (2003)

[89] Mazuyer D, Cayer-Barrioz J, Tonck A, Jarnias F. Friction dynamics of confined weakly adhering boundary layers. Langmuir 24(8): 3857-3866 (2008)

[90] Tománek D, Zhong W, Thomas H. Calculation of an atomically modulated friction force in atomic-force microscopy. Europhys Lett 15(8): 887-892 (1991)

[91] Gnecco E, Bennewitz R, Gyalog T, Loppacher C, Bammerlin M, Meyer E, Güntherodt H J. Velocity dependence of atomic friction. Phys Rev Lett 84(6): 1172-1175 (2000)

[92] Müser M H. Nature of mechanical instabilities and their effect on kinetic friction. Phys Rev Lett 89(22): 224301 (2002)

[93] Riedo E, Gnecco E, Bennewitz R, Meyer E, Brune H. Interaction potential and hopping dynamics governing sliding friction. Phys Rev Lett 91(8): 084502 (2003)

[94] Glosli J N, McClelland G M. Molecular dynamics study of sliding friction of ordered organic monolayers. Phys Rev Lett 70(13): 1960-1963 (1993)

[95] Kong Y C, Tildesley D J, Alejandre J. The molecular dynamics simulation of boundary-layer lubrication. Mol Phys 92(1): 7-18 (1997) 
[96] Chandross M, Grest G S, Stevens M J. Friction between alkylsilane monolayers: Molecular simulation of ordered monolayers. Langmuir 18(22): 8392-8399 (2002)

[97] Zhang L Z, Leng Y S, Jiang S Y. Tip-based hybrid simulation study of frictional properties of self-assembled monolayers: effects of chain length, terminal group, scan direction, and scan velocity. Langmuir 19(23): 9742-9747 (2003)

[98] Chen J Y, Ratera I, Park J Y, Salmeron M. Velocity dependence of friction and hydrogen bonding effects. Phys Rev Lett 96(23): 236102 (2006)

[99] Kapila V, Deymier P A, Raghavan S. Molecular dynamics simulations of friction between alkylsilane monolayers. Modell Simul Mater Sci Eng 14(2): 283-297 (2006)

[100] Ewen J P, Gattinoni C, Morgan N, Spikes H A, Dini D. Non-equilibrium molecular dynamics simulations of organic friction modifiers adsorbed on iron-oxide surfaces. Langmuir 32(18): 4450-4463 (2016)

[101] Müser M. Velocity dependence of kinetic friction in the Prandtl-Tomlinson model. Phys Rev B 84(12): 125419 (2011)

[102] Faraday M. The decomposition of chloride of silver by hydrogen and by zinc. Quart J Liter Sci Arts 8: 374-376 (1820)

[103] Ostwald W. The Fundamental Principles of Chemistry. Morse H W, trans. New York (USA): Longman Green, 1909.

[104] Bell G. Models for the specific adhesion of cells to cells. Science 200(4342): 618-627 (1978)

[105] Beyer M K, Clausen-Schaumann H. Mechanochemistry: the mechanical activation of covalent bonds. Chem Rev 105(8): 2921-2948 (2005)

[106] Boldyrev V V. Mechanochemistry and mechanical activation of solids. Russ Chem Rev 75(3): 177-189 (2006)

[107] Kaupp G. Mechanochemistry: The varied applications of mechanical bond-breaking. Cryst Eng Comm 11(3): 388-403 (2009)

[108] James S L Adams C J, Bolm C, Braga D, Collier P, Friščić T, Grepioni F, Harris K D M, Hyett G, Jones W, et al. Mechanochemistry: Opportunities for new and cleaner synthesis. Chem Soc Rev 41(1): 413-447 (2012)

[109] Ribas-Arino J, Marx D. Covalent mechanochemistry: theoretical concepts and computational tools with applications to molecular nanomechanics. Chem Rev 112(10): 5412-5487 (2012)

[110] Takacs L. The historical development of mechanochemistry. Chem Soc Rev 42(18): 7649-7659 (2013)

[111] Beldon P J, Fábián L, Stein R S, Thirumurugan A, Cheetham A K, Friščić T. Rapid room-temperature synthesis of zeolitic imidazolate frameworks by using mechanochemistry. Angew Chem Int Ed Engl 49(50): 9640-9643 (2010)
[112] Keller D, Bustamante C. The mechanochemistry of molecular motors. Biophys J 78(2): 541-556 (2000)

[113] Greenberg M J, Moore J R. The molecular basis of frictional loads in the in vitro motility assay with applications to the study of the loaded mechanochemistry of molecular motors. Cytoskeleton 67(5): 273-285 (2010)

[114] Lenhardt J M, Ogle J W, Ong M T, Choe R, Martinez T J, Craig S L. Reactive cross-talk between adjacent tensiontrapped transition states. J Am Chem Soc 133(10): 3222-3225 (2011)

[115] Craig S L. Mechanochemistry: A tour of force. Nature 487(7406): 176-177 (2012)

[116] Knothe Tate M L, Gunning P W, Sansalone V. Emergence of form from function-Mechanical engineering approaches to probe the role of stem cell mechanoadaptation in sealing cell fate. BioArchitecture 6(5): 85-103 (2016)

[117] Zhu C. Mechanochemitry: a molecular biomechanics view of mechanosensing. Ann Biomed Eng 42(2): 388-404 (2014)

[118] Black A L, Lenhardt J M, Craig S L. From molecular mechanochemistry to stress-responsive materials. J Mater Chem 21(6): 1655-1663 (2011)

[119] Brantley J N, Wiggins K M, Bielawski C W. Polymer mechanochemistry: the design and study of mechanophores. Polym Int 62(1): 2-12 (2013)

[120] De Simo M, Hilmer F B. Process for stabilizing polymers. U.S. Patent 2085525, Jun. 1937.

[121] Morris W J, Schnurmann R. Mechanical degradation of large molecules. Nature 16(4072): 674 (1947)

[122] Marx N, Ponjavic A, Taylor R I, Spikes H A. Study of permanent shear thinning of VM polymer solutions. Tribol Lett 65(3): 106 (2017)

[123] Sohma J. Mechanochemistry of polymers. Prog Polym Sci 14(4): 451-596 (1989)

[124] De Gennes P G. Coil-stretch transition of dilute flexible polymers under ultrahigh velocity gradients. $J$ Chem Phys 60(12): 5030-5042 (1974)

[125] Odell J A, Keller A, Rabin Y. Flow-induced scission of isolated macromolecules. J Chem Phys 88(6): 4022-4028 (1988)

[126] Odell J A, Muller A J, Narh K A, Keller A. Degradation of polymer solutions in extensional flows. Macromolecules 23(12): 3092-3103 (1990)

[127] Gosvami N N, Bares J A, Mangolini F, Konicek A R, Yablon D G, Carpick R W. Mechanisms of antiwear tribofilm growth revealed in situ by single-asperity sliding contacts. Science 348(6230): 102-106 (2015)

[128] Adams H L, Garvey M T, Ramasamy U S, Ye Z J, Martini A, Tysoe W T. Shear-induced mechanochemistry: pushing molecules around. J Phys Chem C 119(13): 7115-7123 (2015) 
[129] Adams H, Miller B P, Kotvis P V, Furlong O J, Martini A, Tysoe W T. In situ measurements of boundary film formation pathways and kinetics: dimethyl and diethyl disulfide on copper. Tribol Lett 62(1): 12 (2016)

[130] Felts J R, Oyer A J, Hernández S C, Whitener Jr K E, Robinson J T, Walton S G, Sheehan P E. Direct mechanochemical cleavage of functional groups from graphene. Nat Commun 6: 6467 (2015)

[131] Yeon J, He X, Martini A, Kim S H. Mechanochemistry at solid surfaces: polymerization of adsorbed molecules by mechanical shear at tribological interfaces. ACS Appl Mater Interfaces 9(3): 3142-3148 (2017)

[132] He X, Kim S H. Mechanochemistry of physisorbed molecules at tribological interfaces: molecular structure dependence of tribochemical polymerization. Langmuir 33(11): 2717-2724 (2017)

[133] Brizmer V, Matta C, Nedelcu I, Morales-Espejel G E. The influence of tribolayer formation on tribological performance of rolling/sliding contacts. Tribol Lett 65(2): 57 (2017)

[134] Akchurin A, Bosman R. A deterministic stress-activated model for tribo-film growth and wear simulation. Tribol Lett 65(2): 59 (2017)

[135] Zhang J, Spikes H. On the mechanism of ZDDP antiwear film formation. Tribol Lett 63(2): 24 (2016)

[136] Dickinson J T, Park N S, Kim M W, Langford S C. A scanning force microscope study of a tribochemical system: stress-enhanced dissolution. Tribol Lett 3(1): 69-80 (1997)

[137] Kopta S, Salmeron M. The atomic scale origin of wear on mica and its contribution to friction. J Chem Phys 113(18): 8249-8252 (2000)

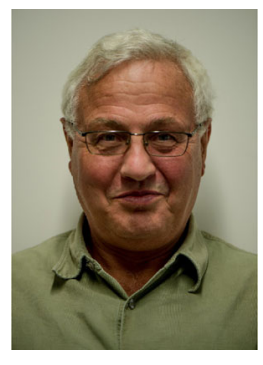

Hugh SPIKES. He graduated in natural sciences from the University of Cambridge in 1968 and received his PhD degree in mechanical engineering from the University of London in 1972. He joined the staff of the Mechanical Engineering Department at Imperial College in
[138] Gotsmann B, Lantz M A. Atomistic wear in a single asperity sliding contact. Phys Rev Lett 101(12): 125501 (2008)

[139] Jacobs T D B, Carpick R W. Nanoscale wear as a stressassisted chemical reaction. Nat Nanotechnol 8(2): 108-112 (2013)

[140] Ewen J P, Gattinoni C, Morgan N, Spikes H A, Dini D. Nonequilibrium molecular dynamics simulations of organic friction modifiers adsorbed on iron oxide surfaces. Langmuir 32(18): 4450-4463 (2016)

[141] Mott N F. Slip at grain boundaries and grain growth in metals. Proc Phys Soc 60(4): 391-394 (1948)

[142] Gnecco E, Bennewitz R, Socoliuc A, Meyer E. Friction and wear on the atomic scale. Wear 254(9): 859-862 (2003)

[143] Konda S S M, Brantley J N, Bielawski C W, Makarov D E. Chemical reactions modulated by mechanical stress: extended Bell theory. J Chem Phys 135(16): 164103 (2011)

[144] Furlong O J, Manzi S J, Martini A, Tysoe W T. Influence of potential shape on constant-force atomic-scale sliding friction models. Tribol Lett 60(2): 21 (2015)

[145] Tysoe W. On stress-induced tribochemical reaction rates. Tribol Lett 65(2): 48 (2017)

[146] Zhang J, Tan A, Spikes H. Effect of base oil structure on elastohydrodynamic friction. Tribol Lett 65(1): 13 (2017)

[147] Galmiche B, Ponjavic A, Wong J S S. Flow measurements of a polyphenyl ether oil in an elastohydrodynamic contact. J Phys Condens Matter 28(13): 134005 (2016)

[148] Wool R S, Bretzlaff R Y, Li B H, Wang C H, Boyd R H. Infrared and Raman spectroscopy of stressed polyethylene. J Polym Sci Part B Polym Phys 24(5): 1039-1066 (1986)

1978, where in 1996 he became professor and head of the Tribology Group. He is currently a senior research fellow at Imperial College. His research areas cover all aspects of liquid lubrication, from hydrodynamic to boundary, with a particular interest in the influence of lubricant molecular composition on performance. He is a recipient of the ASME Mayo D Hersey Award, STLE International Award, and the Tribology Gold Medal. 